\title{
DATA ON THE DISTRIBUTION OF SELECTED SORICOMORPHA AND RODENTIA SPECIES IN POLAND
}

\author{
Agata BanaszeK*, WŁodzimierz Chętnicki, StanisŁaW \\ FEDYK, Katarzyna A. JADWiszczak and PaWeE Mirski
}

Institute of Biology, University of Białystok; Świerkowa 20B, 15-950 Białystok, Poland; e-mail: banaszek@uwb.edu.pl, tel: +48 857457290

*Corresponding author

\begin{abstract}
We present current data on the occurrence of four Soricomorpha and 15 Rodentia species. The mammals were collected during trapping of common shrews and yellow-necked mice for chromosomal research and the common hamsters for phylogeography and genetic variability research. The maps of species occurrence were created in ArcGIS and each map is accompanied by the list of localities with geographic coordinates and year of capture.
\end{abstract}

Key words: species distribution, maps of occurrence, lists of localities, GIS

\section{INTRODUCTION}

The pattern of distribution is not permanent for a species. Numerous factors affect the distribution and the species have to adjust their ranges to changing environments. It is likely that the Quaternary epoch was the richest in events strongly influencing the abundance and distribution of species (WILLIS and Niklas 2004). The Pleistocene climate oscillations have been shown to dramatically change species distributions. On the other hand, climate change over the last 100 years has already caused the shifts in species distribution and abundances (Root et al. 2003). The biotic factors, as for example invasive species, also have a significant impact on native fauna distribution (MoONEY and Cleland 2001). Currently, anthropogenic changes, which cause fragmenta- 
tion and destruction of habitats, very strongly reduce species ranges. Hence, it is necessary to update knowledge on the distribution of species and record the changes.

In Poland, the distribution of mammal species was compiled in the Atlas of Polish Mammals (Pucek and Raczyński 1983). Since that time, the ranges of some species changed considerably, for example that of the common hamster, which has lost $75 \%$ of its distribution (ZIOMEK and BANASZeK 2007). Hence, it is of great value, that the Institute of Nature Conservation PAS, Department of Systematic Zoology UAM, and Museum and Institute of Zoology PAS started in 2008 the initiative to bring up to date the Atlas of Polish Mammals and digitize the information on the distribution of the Polish theriofauna (OKARMA 2009).

Our group has worked for several decades on various projects of population genetics of multiple mammal species. Mainly, we were interested in the chromosomal variation of the common shrew (FEDYK 1995), the B chromosome polymorphism in yellow-necked mice (BANASZEK and JADWISZCZAK 2006) and molecular diversity of common hamster populations (BANASZEK et al. 2010). All these topics required the capture of animals. Moreover, we took part in several monitoring programmes, for example the state of theriofauna in the Knyszyńska Forest. We usually used live traps in which many other species were captured, not only those necessary for our research. In effect, we collected a large database on incidence of several Soricomorpha and over a dozen Rodentia species. These data do not form full distribution maps, as our research followed mainly the distribution of the common shrew chromosomal hybrid zones.

The aim of this paper is to enlarge our knowledge on the current occurrence of some Soricomorpha and Rodentia species. We decided to create maps with the lists of locations and geographic coordinates, which are ready to use for the purposes of a new Atlas of Polish Mammals.

\section{MATERIAL AND METHODS}

The capture of small mammals for cytogenetic study of the common shrew was performed during the years 1980-2008. The large or medium pitfalls (metal cones) were used. Common shrews were sacrificed for cytogenetic preparations, and all the other specimens were released. The identification of a species was done directly at the trapping site on the basis of morphological traits. As the pitfalls may trap many individuals at the same time, the death of some individuals is unavoidable. The dead animals were taken to the lab for more precise species determination, by use of morphometry and dentition features.

The collection of yellow-necked mice was conducted during the years 2000 -2005 using Białowieża wooden box-traps. Common hamster research was performed in years 2006-2011. The hamsters were collected in large live traps. Small mammals are rarely able to close the hamster traps and even then, they often get out between the wires, hence, we used only the data of rats captured in hamster 
traps. The common hamster itself was excluded from this study as the changes in its distribution have been described previously (ZiomeK and BANASZEK 2007).

The habitats we collected animals from were chosen for our specific purposes. For example, for shrews we looked for wet habitats, for yellow-necked mice we chose forests with rich undergrowth, and for common hamsters agricultural land used for wheat or sugar beet cultivation with good soil. This means, that even in areas where our research was very intensive, we cannot exclude the presence of species we did not captured.

Our trapping lines were usually named after the closest village or city. All locations were found in the interactive map of Poland (www.mapa.szukacz.pl), which provides the geographic coordinates of places in WGS 84 system. We used ArcGIS 10.0 to plot our localities into UTM 10 x $10 \mathrm{~km}$ grids. The squares which contained localities of a species were marked with a point. A point in a square does not represent a single locality, it may cover a few sites of occurrence. Empty squares indicate a lack of data. Each map is accompanied by the list of localities with geographic coordinates and year of capture (Appendix 1). The authors will send on the request the list of localities for particular species in Excel format, which is easy to import into ArcGIS.

\section{RESULT}

Soricomorpha - There are four species in our database. The lists of localities are given in Appendix 1.

\section{European mole (Talpa europaea Linnaeus, 1758)}

Collected incidentally in our research, as it both leads subterranean life and can easily get out of pitfall traps.

\section{Common shrew (Sorex araneus Linnaeus, 1758)}

The most numerous species in our database, as most of our trapping effort was connected with collecting shrews. The species determination is without doubt as most of our shrews were karyotyped. The distribution of the common shrew is the only case which can be used as an evidence for the lack of other species i.e. the masked shrew, Sorex caecutiens Laxmann, 1788. Many of our trapping expeditions were performed in northeastern Poland close to the Białowieża Forest which forms the western distribution edge of S. ceacutiens in Poland. We were always aware of the possibility of finding a masked shrew locality. However, even dubious for us specimens on the basis of morphometry, always proved to be $S$. araneus on the basis of karyotype.

\section{Eurasian pygmy shrew (Sorex minutus Linnaeus, 1766)}

The Eurasian pygmy shrews were collected in similar habitats to those of the common shrews, hence they were also numerous in this database. Species determination is reliable on the basis of external traits and external morphometry. 
Talpa europaea

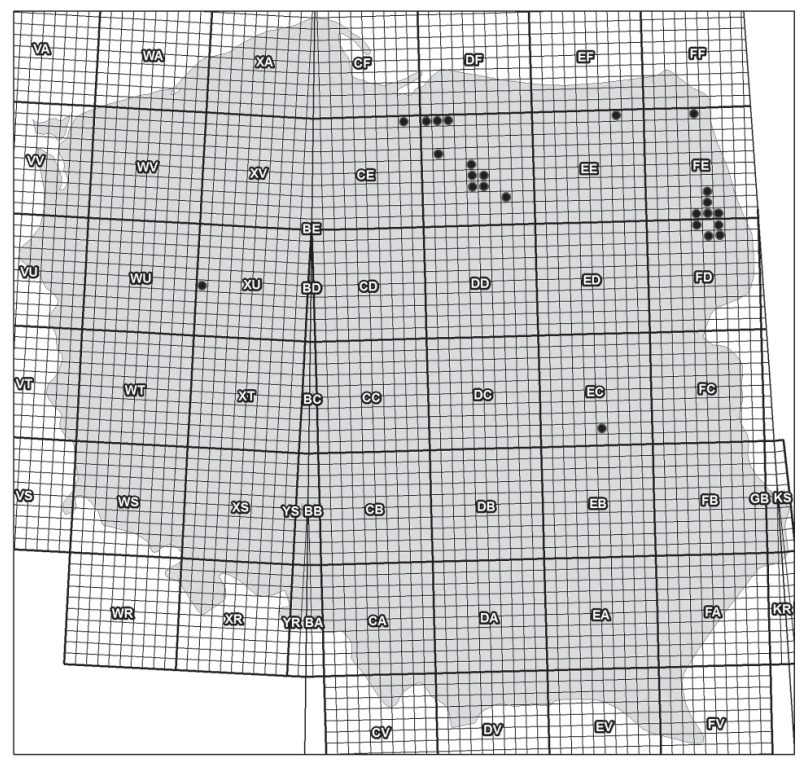

Figure 1. Map of the occurrence of the European mole (Talpa europaea).

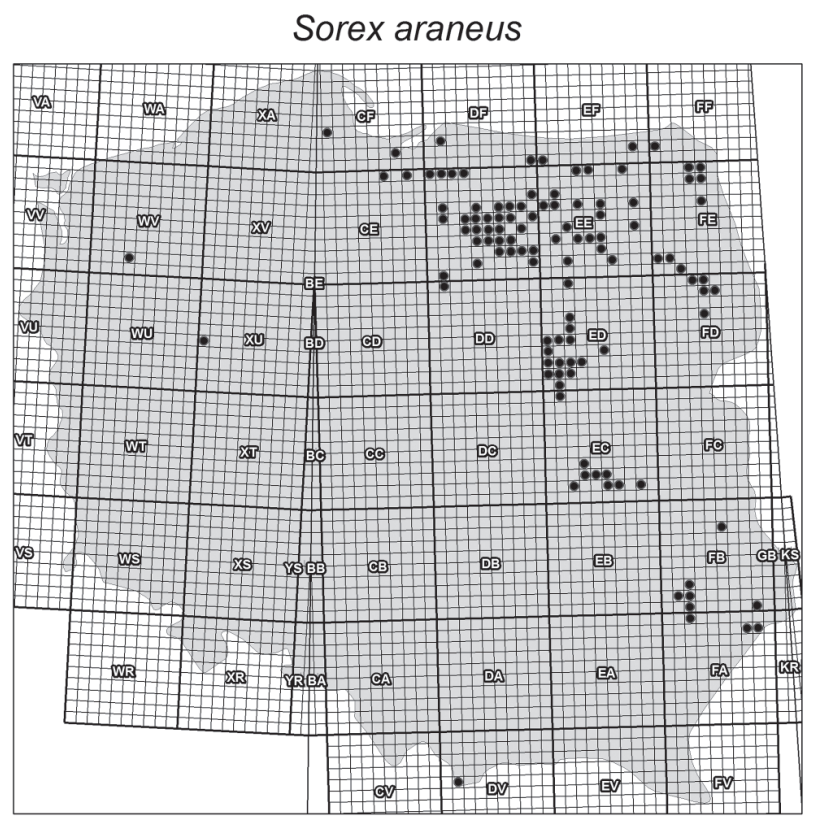

Figure 2. Map of the occurrence of the common shrew (Sorex araneus). 


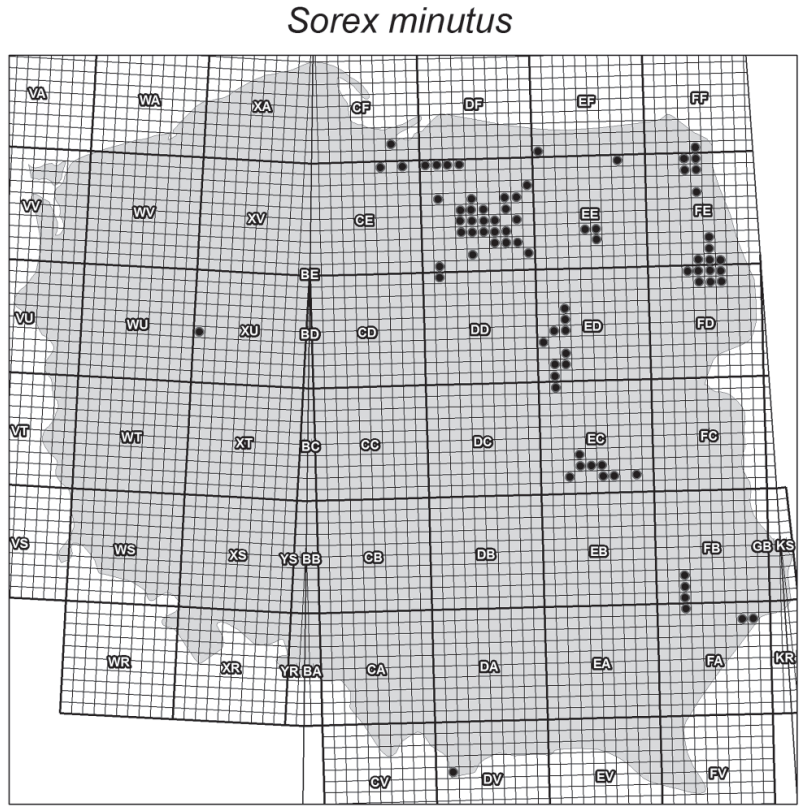

Figure 3. Map of the occurrence of the Eurasian pygmy shrew (Sorex minutus).

\section{Eurasian water shrew (Neomys fodiens (Pennant, 1771))}

The species determination is certain on the basis of external traits and morphometry.

Rodentia - There are fifteen species in our database. The lists of localities are given in Appendix 1.

Bank vole (Myodes glareolus Schreber, 1780)

The bank vole was common in our research. The species was determined usually on external morphology, sometimes confirmed using dentition features.

\section{Water vole (Arvicola terrestris (Linnaeus, 1758))}

Collected by us quite rarely, as large water voles are usually able to jump out of the pitfalls.

European pine vole (Pitymys subterraneus (de Selys - Longchamps, 1836))

Collected incidentally in our research because of their subterranean way of life. Species was determined on the external morphological traits and some dead individuals were verified on the basis of teeth characteristics. 


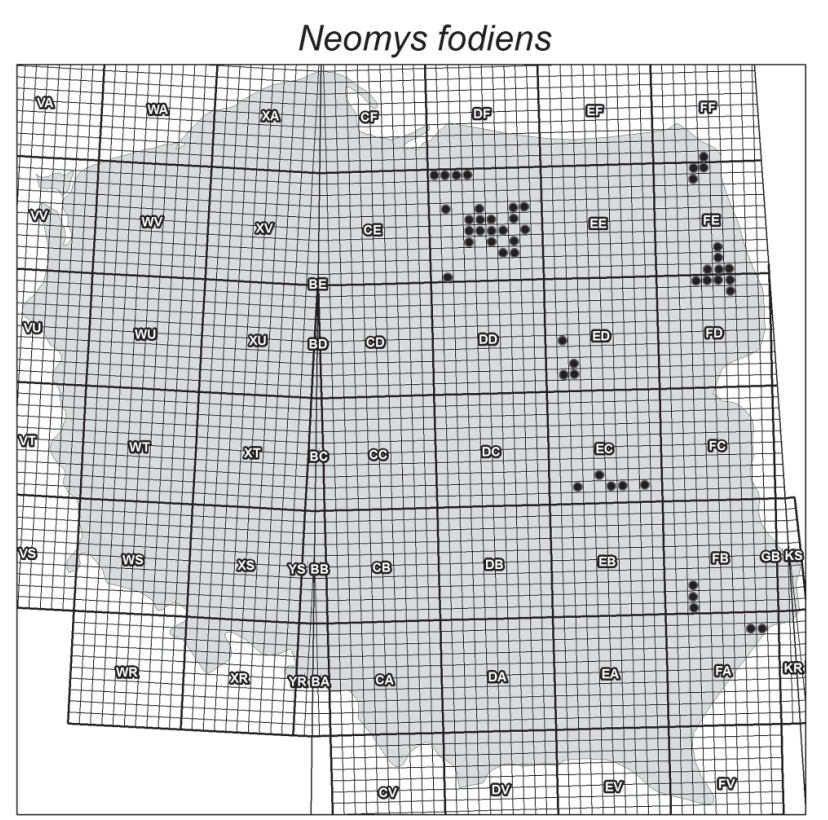

Figure 4. Map of the occurrence of the Eurasian water shrew (Neomys fodiens).

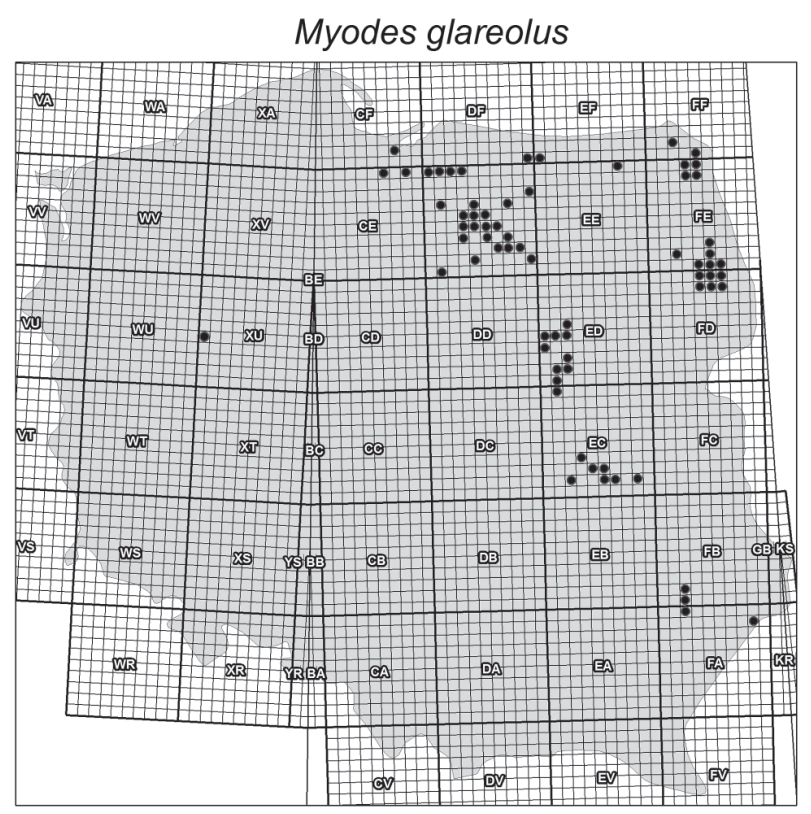

Figure 5. Map of the occurrence of the bank vole (Myodes glareolus). 


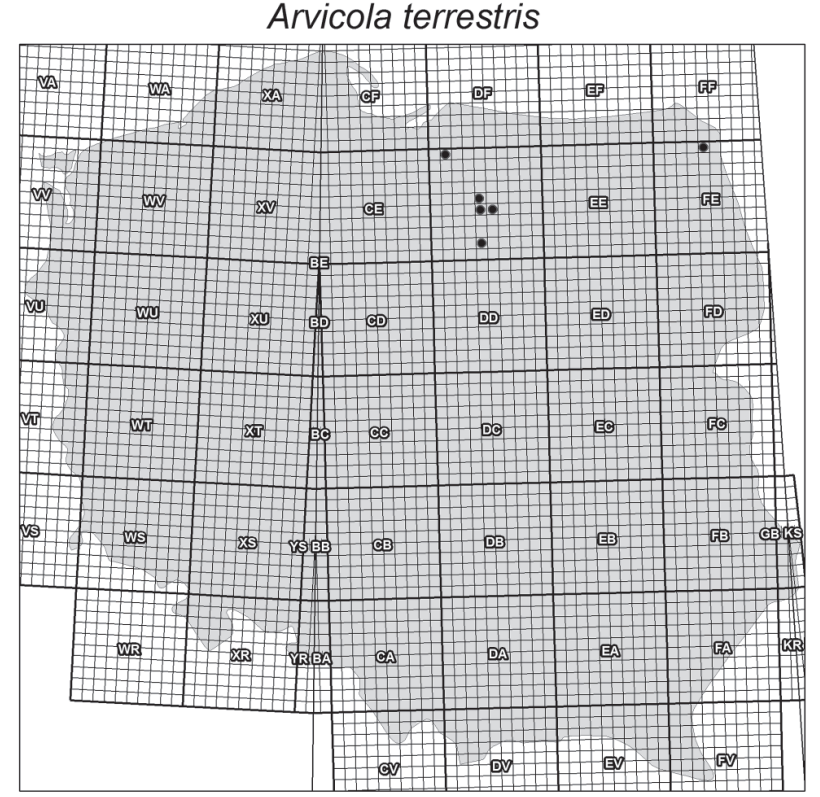

Figure 6. Map of the occurrence of the water vole (Arvicola terrestris).

\section{Pitymys subterraneus}

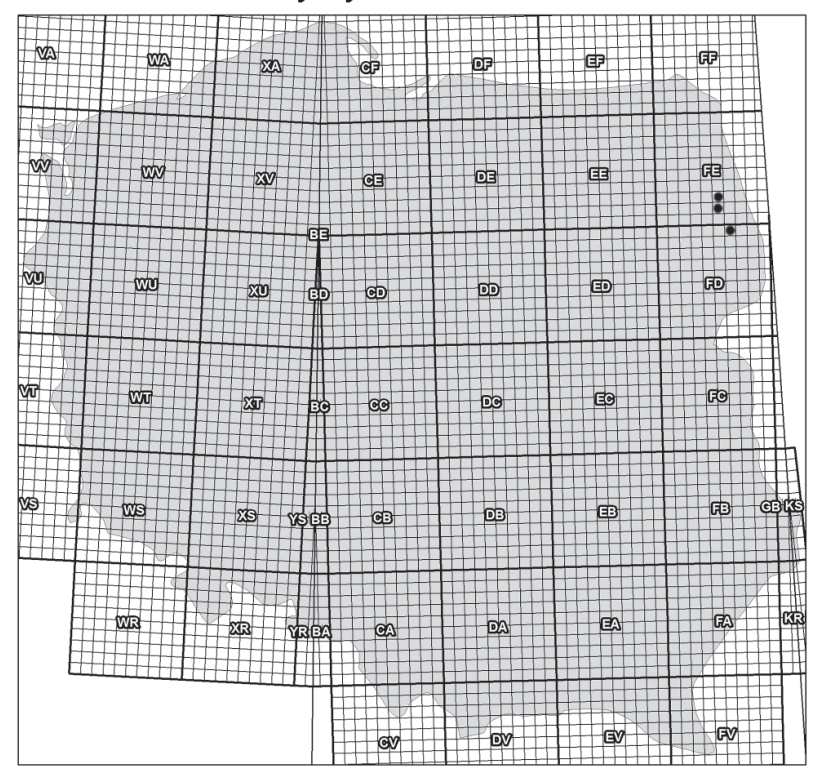

Figure 7. Map of the occurrence of the European pine vole (Pitymys subterraneus). 
Microtus sp.

Most of Microtus specimens collected during our research were released without species determination, as dependable identification is not possible on external traits alone. Species determination was conducted for dead animals on the basis of dentition features. That is why the localities of Microtus sp. are quite scarce in this study, although the voles were numerous in the traps. Three species were recorded in our database: the field vole (Microtus agrestis (Linnaeus, 1761)), the common vole (Microtus arvalis (Pallas, 1779)) and the root vole (Microtus oeconomus (Pallas, 1776)).

House mouse (Mus musculus Linnaeus, 1758)

Collected by us sporadically as we rarely tried to collect animals close to human habitation, hence we probably trapped only migrating individuals.

\section{Common rat (Rattus norvegicus (Berkenhout, 1769))}

The rats were captured in the common hamster traps. The species determination was confirmed by molecular marker - the control region of mtDNA (Banaszek, unpubl. data). A dead animal found in the fields of Pilaszkowice was genotyped and proved to be the common rat by comparison with the sequences in GenBank. Moreover, two rats were collected in large pitfalls in the Knyszyńska Forest.

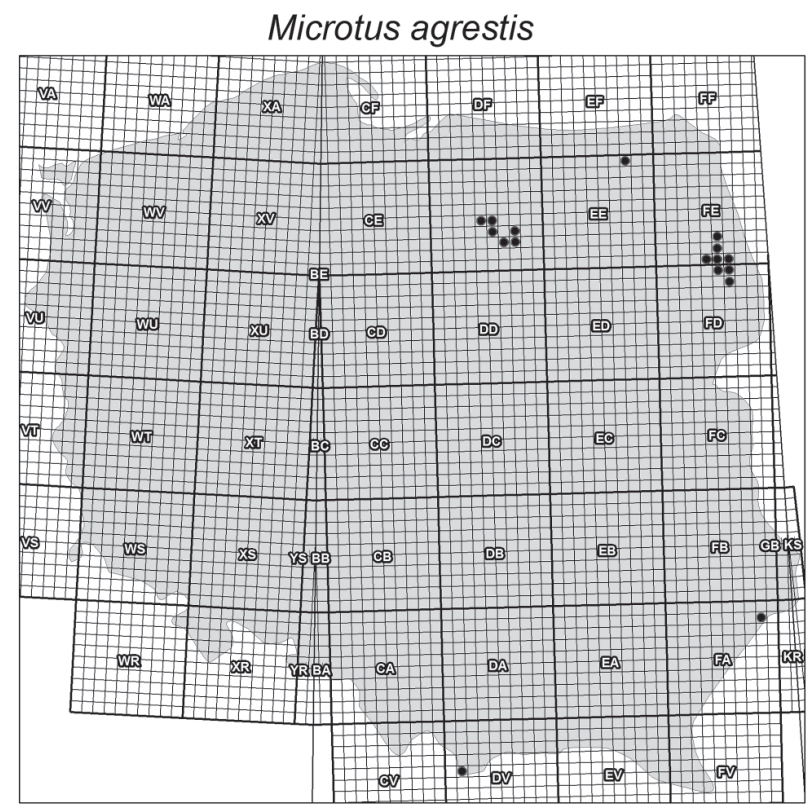

Figure 8. Map of the occurrence of the field vole (Microtus agrestis). 


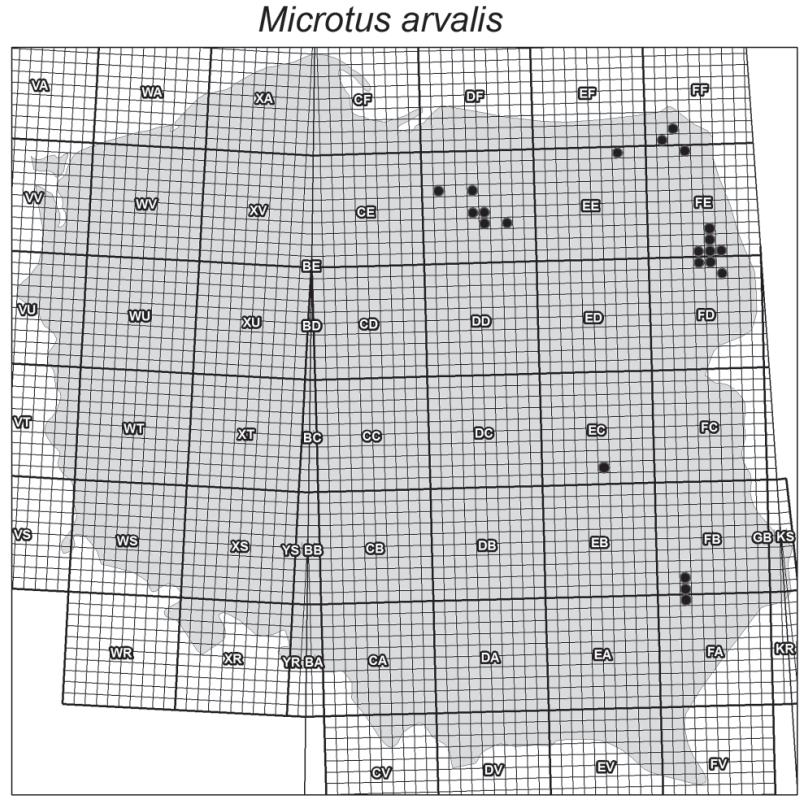

Figure 9. Map of the occurrence of the common vole (Microtus arvalis).

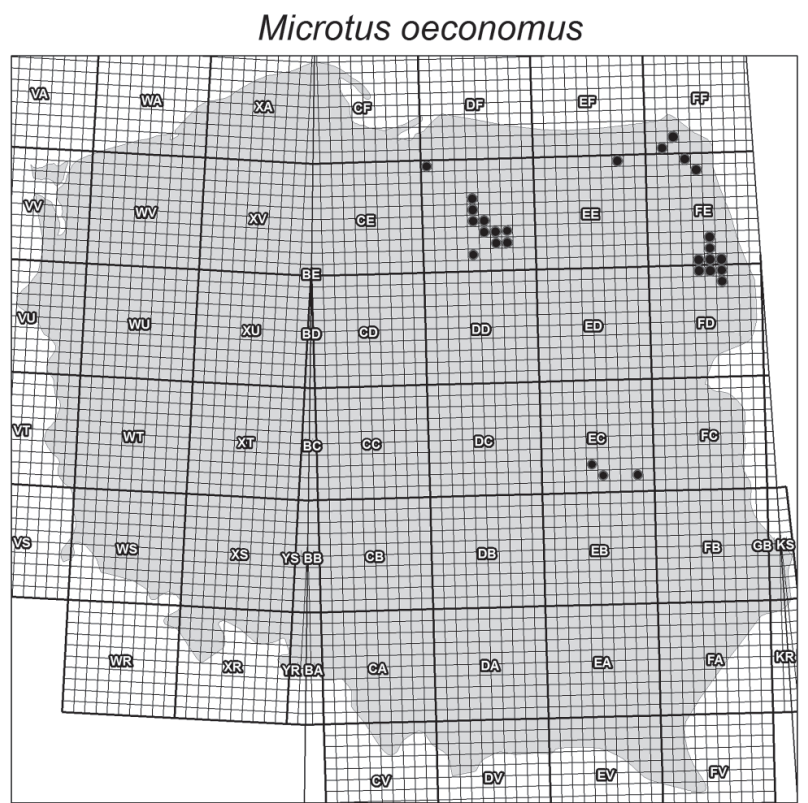

Figure 10. Map of the occurrence of the root vole (Microtus oeconomus). 


\section{Mus musculus}

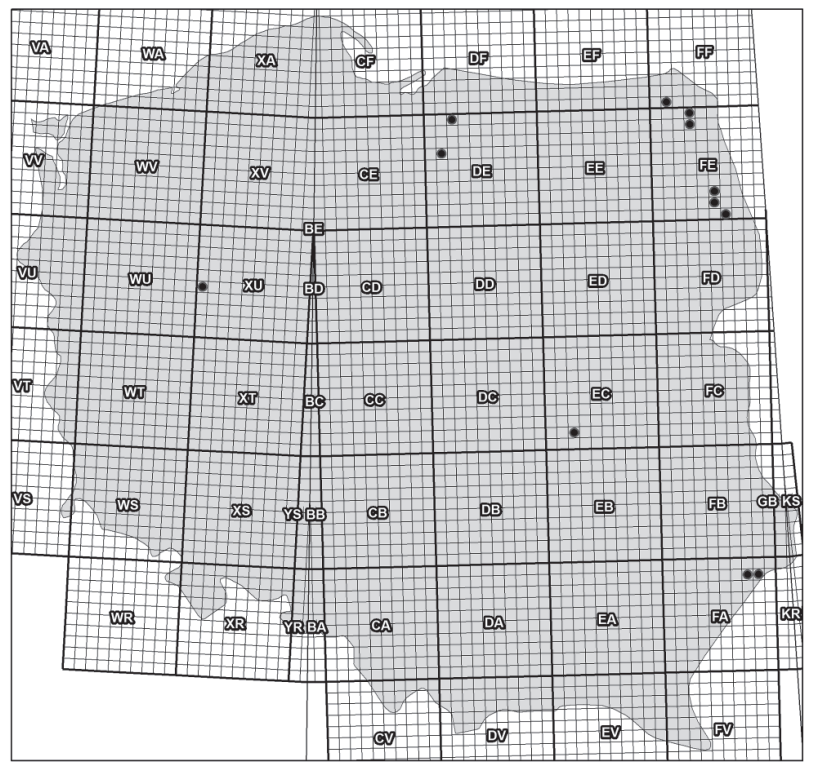

Figure 11. Map of the occurrence of the house mouse (Mus musculus).

Rattus norvegicus

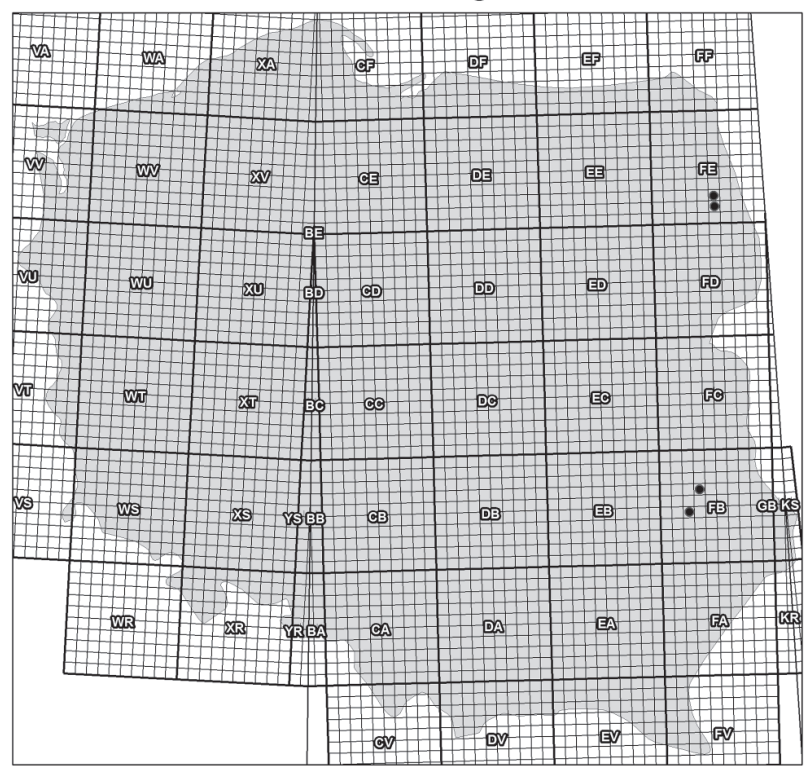

Figure 12. Map of the occurrence of the common rat (Rattus norvegicus). 
Harvest mouse (Micromys minutus (Pallas, 1771))

Harvest mice are easy to recognize by external traits. Although we have listed quite a number of localities, the harvest mice were infrequent in our traps, as they usually move above ground level on grass stalks and bush branches.

\section{Apodemus sp.}

The yellow-necked mouse (Apodemus flavicollis (Melchior, 1834)) and the wood mouse (Apodemus sylvaticus (Linnaeus, 1758)) are difficult to discriminate. Colour traits usually allow doubtless identification of adult specimens of the yellow-necked mouse. However, young A. flavicollis can be easily mistaken with the smaller wood mouse. Hence, we usually released most Apodemus mice without species determination. During research for B chromosomes in Apodemus, the species determination was done on the basis of LDH (lactate dehydrogenase) electrophoresis or C-banded karyotypes (Engel et al., 1973). Any A. sylvaticus determinations and locations in our maps were done during $\mathrm{B}$ chromosome research. The striped field mouse (Apodemus agrarius (Pallas, 1771)) is distinct from the two former species of Apodemus due to the presence of a black dorsal stripe. However, because of this stripe, it can sometimes be mistaken for Sicista betulina (Pallas, 1778). As we were aware of this, some cases of young A. agrarius/ S. betulina were excluded.

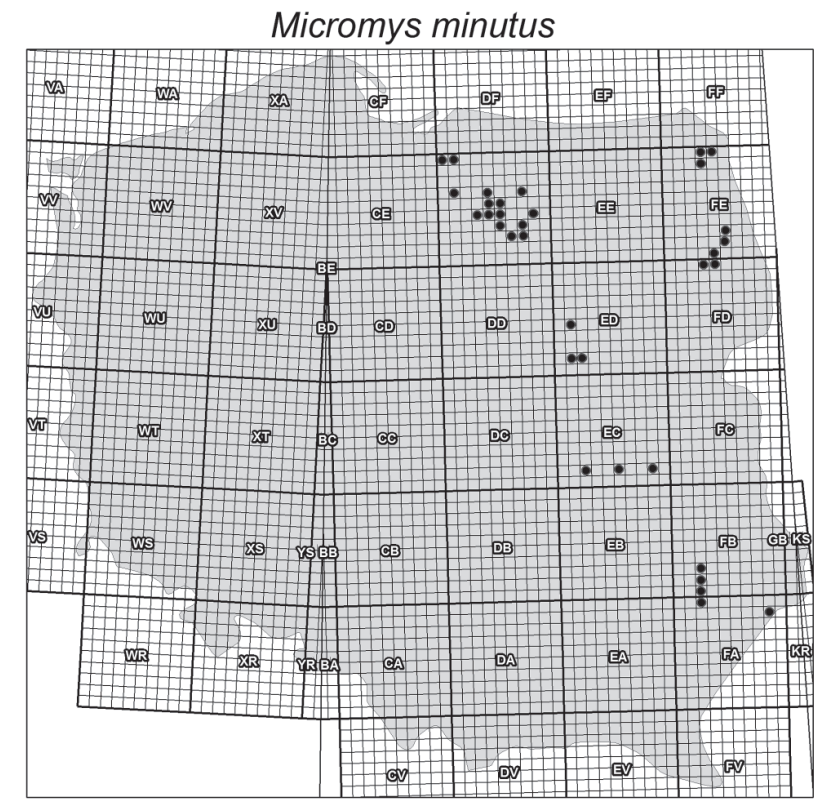

Figure 13. Map of the occurrence of the harvest mouse (Micromys minutus). 
Apodemus flavicollis

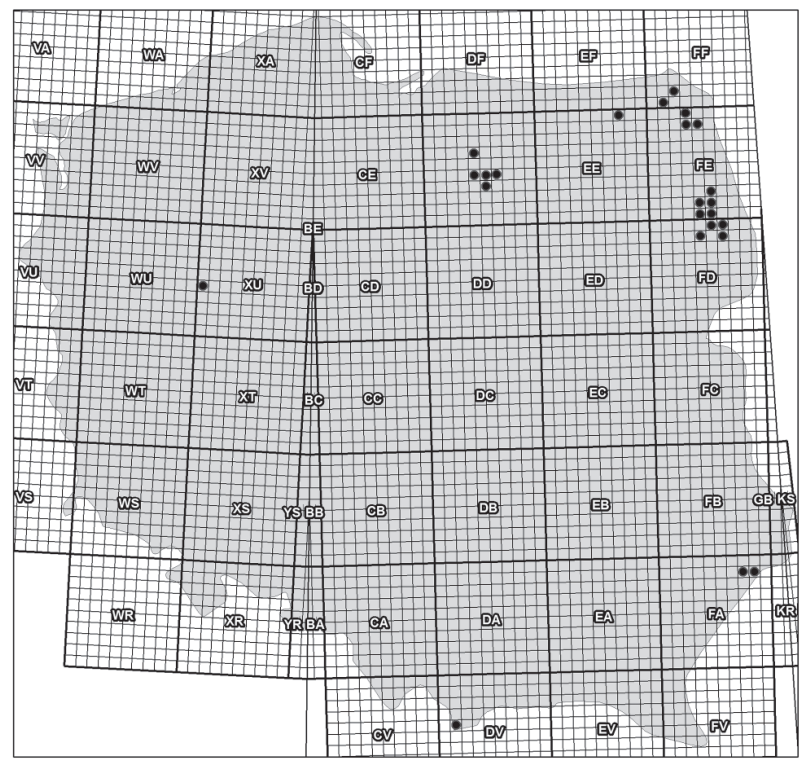

Figure 14. Map of the occurrence of the yellow-necked mouse (Apodemus flavicollis).

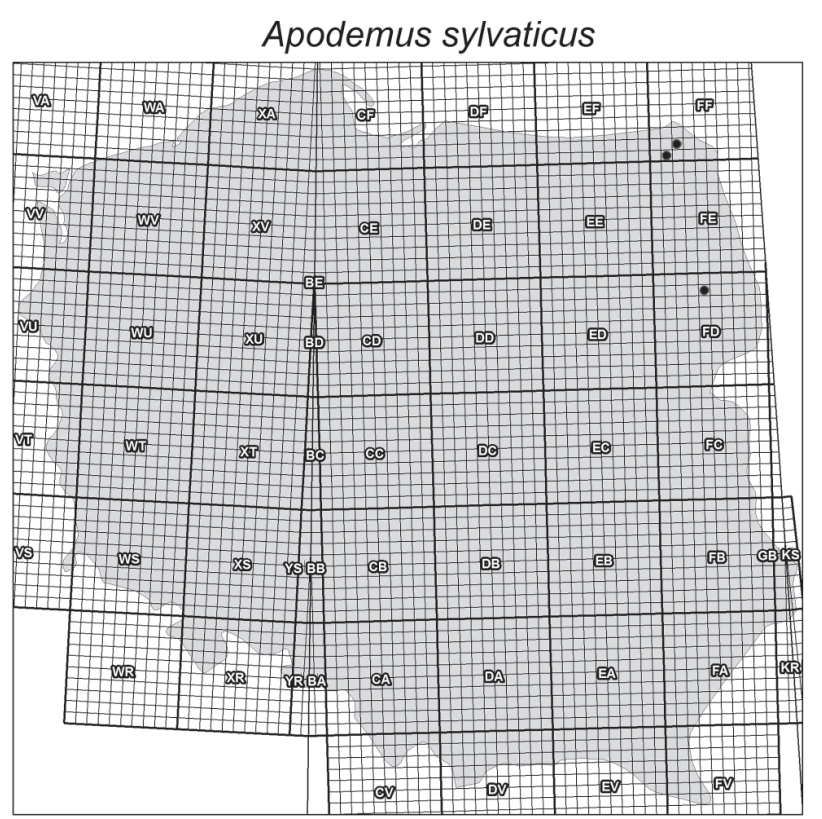

Figure 15. Map of the occurrence of the wood mouse (Apodemus sylvaticus). 
Apodemus agrarius

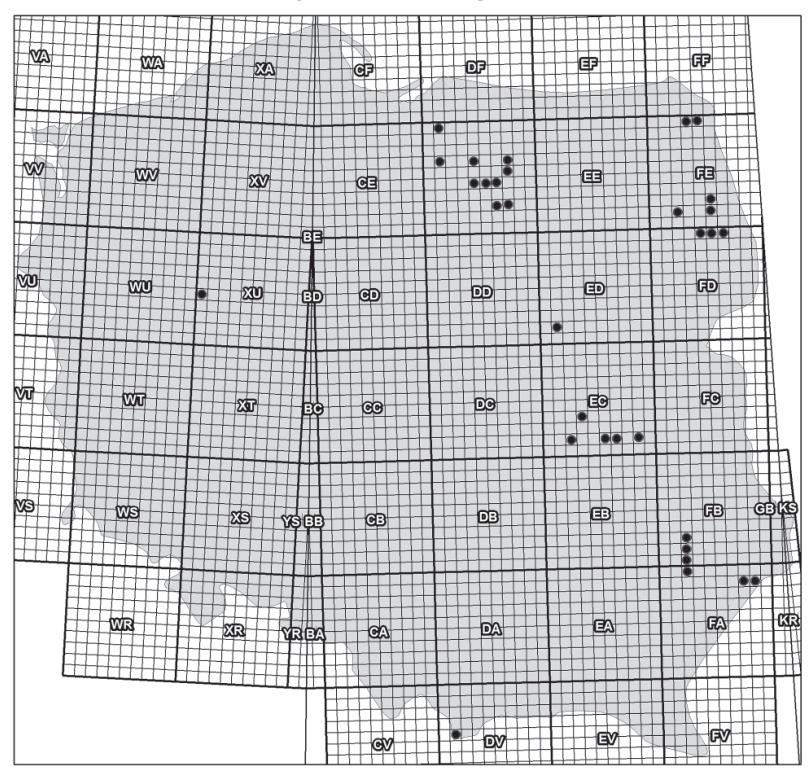

Figure 16. Map of the occurrence of the striped field mouse (Apodemus agrarius)

Sicista betulina

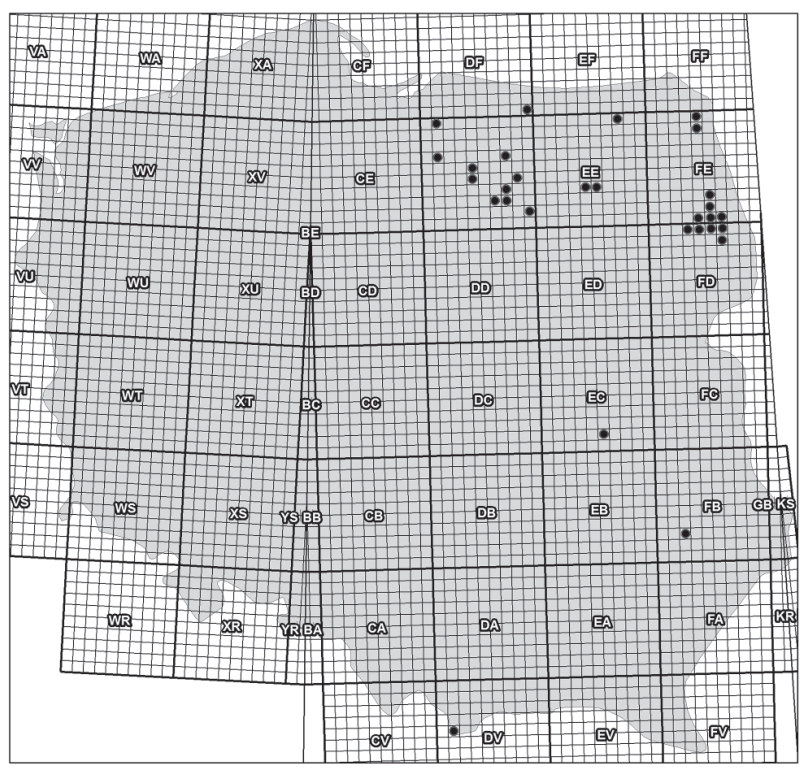

Figure 17. Map of the occurrence of the northern birch mouse (Sicista betulina). 
Northern birch mouse (Sicista betulina (Pallas, 1778))

Species was determined on the basis of external traits and morphometry, additionally some individuals were karyotyped (FEDYk et al., 2011). Our localities in Masuria in DE square are located a little bit more to the west than in the formerly described distribution of S. betulina.

Forest dormouse (Dryomys nitedula (Pallas, 1779)) and the common dormouse (Muscardinus avellanarius (Linnaeus, 1758))

The dormice were collected very rarely as we never set traps on trees. The dormice move among branches of shrubs, hence the animals collected on the ground were probably looking for underground, winter refuge. For the forest dormouse, we present the localities in the Knyszyńska Forest. No sites of occurrence of $D$. nitedula from the Knyszyńska Forest were shown in the Atlas of Polish Mammals (PUCEK and RACZYŃski, 1983), although such localities were reported in FE UTM square (BIAŁAs et al., 1982). Here we report two more sites for the forest dormouse from the Knyszyńska Forest.

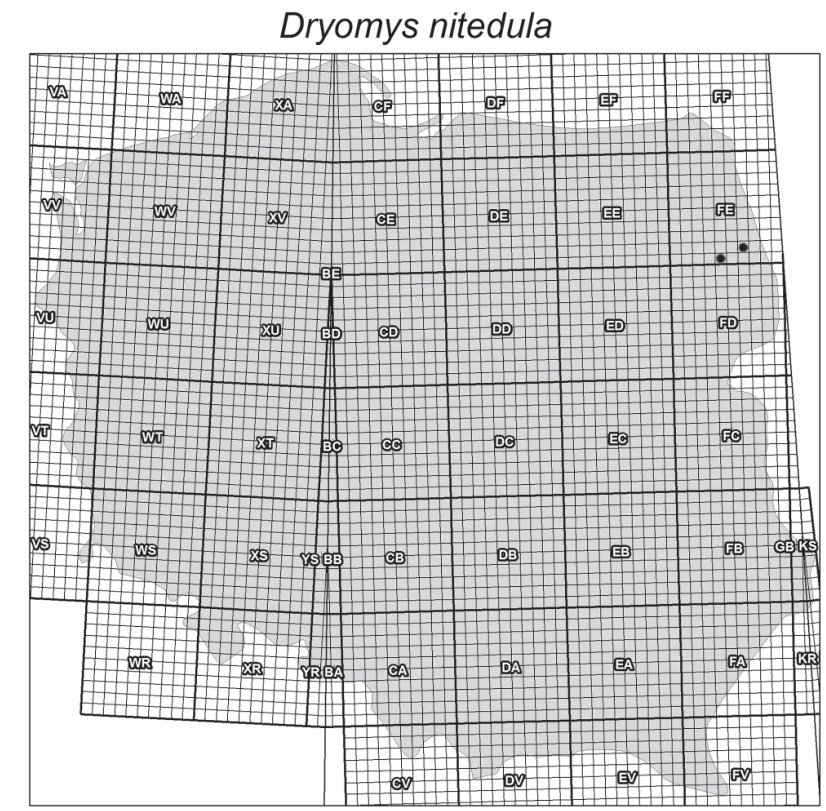

Figure 18. Map of the occurrence of the forest dormouse (Dryomys nitedula). 


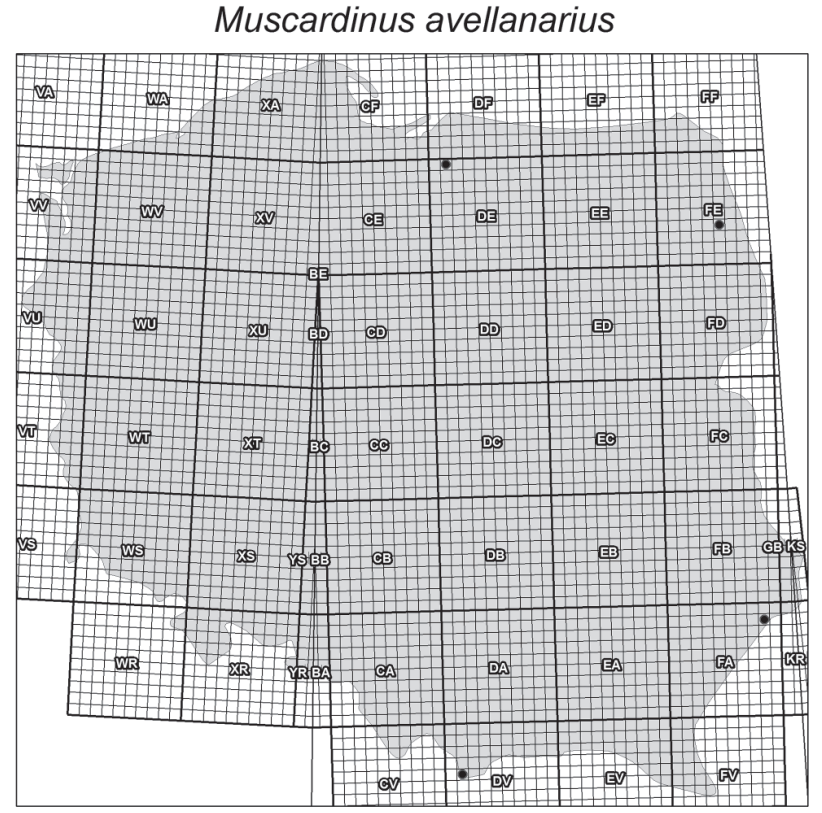

Figure 19. Map of the occurrence of the common dormouse (Muscardinus avellanarius).

\section{CONCLUSIONS}

In this study, we presented data on the occurrence of four Soricomorpha and 15 Rodentia species. Most of the collected species occur over the whole of Poland and our localities are therefore within the formerly described ranges. Although some of our points are new for a particular species, it is only the result of intense research in certain areas, and not changes in distribution or abundance of the species. In two cases, that of the northern birch mouse and the forest dormouse, our points of occurrence are outside the former species range (PuCEK and RACZYŃSki 1983). However, in both cases the new localities are close to the formerly described distribution areas. As we do not have data to create full distribution maps, we cannot judge if these new points are the result of extended species ranges or just the result of less intense study in some areas of the Knyszyńska Forest and Masuria during preparation of the former Atlas of Polish Mammals.

\section{Acknowledgements}

The authors wish to express their gratitude to M.Sc Edyta Żuk-Kempa, Dr Janusz Kupryjanowicz and numerous students of the University of Białystok, who took part in our field expeditions. 
APPENDIX 1. LISTS OF LOCALITIES OF SELECTED SORICOMORPHA AND RODENTIA SPECIES IN POLAND.

Table 1. List of the European mole (Talpa europaea) localities.

\begin{tabular}{|c|c|c|c|c|c|}
\hline \multicolumn{2}{|c|}{ UTM square } & \multirow{2}{*}{$\begin{array}{l}\text { Localities } \\
\text { Lubstowo }\end{array}$} & \multicolumn{2}{|c|}{ Geographic coordinates } & \multirow{2}{*}{$\begin{array}{c}\text { Year of capture } \\
1994\end{array}$} \\
\hline $\mathrm{CE}$ & 89 & & 54.131851 & 19.19815 & \\
\hline \multirow[t]{17}{*}{ DE } & 16 & Kupin & 53.844166 & 19.63652 & 1993, 1999 \\
\hline & 19 & Kajmy & 54.088612 & 19.72195 & 1992 \\
\hline & 19 & Pasłęk & 54.066826 & 19.66181 & 1992,1993 \\
\hline & 19 & Winiary & 54.125504 & 19.71557 & 1993 \\
\hline & 29 & Bielica & 54.085045 & 19.81677 & 1992 \\
\hline & 43 & Rychnowska Wola & 53.601521 & 20.12411 & 1991 \\
\hline & 44 & Guzowy Piec & 53.687389 & 20.21625 & 1994 \\
\hline & 45 & Podlejki & 53.730518 & 20.19109 & 1994 \\
\hline & 45 & Worliny & 53.779686 & 20.13292 & 1991 \\
\hline & 53 & Jemiołowo & 53.561832 & 20.29932 & 1990 \\
\hline & 53 & Lutynowo & 53.551113 & 20.34409 & 1994 \\
\hline & 54 & Mańki & 53.664074 & 20.25555 & 1988,1989 \\
\hline & 54 & Miodówko & 53.645294 & 20.37587 & 1991 \\
\hline & 54 & Mycyny & 53.639053 & 20.267 & 1994 \\
\hline & 72 & Natać Wielka & 53.511272 & 20.57244 & 2001 \\
\hline & 72 & Zimna Woda & 53.45215 & 20.6002 & 2002 \\
\hline & 09 & Łączna & 54.079723 & 19.5825 & 2001 \\
\hline $\mathrm{EC}$ & 51 & Stężyca & 51.582561 & 21.77271 & 1997 \\
\hline $\mathrm{EE}$ & 79 & Czerwony Dwór & 54.130104 & 22.18694 & 1984 \\
\hline \multirow[t]{4}{*}{ FD } & 49 & Jurowce & 53.19661 & 23.15286 & 1993 \\
\hline & 58 & Sobolewo & 53.111221 & 23.26858 & 1996 \\
\hline & 68 & Żednia & 53.090096 & 23.46989 & 1983 \\
\hline & 69 & Surażkowo & 53.211678 & 23.45654 & 1983 \\
\hline \multirow[t]{6}{*}{$\mathrm{FE}$} & 40 & Karczmisko & 53.299759 & 23.18604 & 1983 \\
\hline & 49 & Żubrówka & 54.081299 & 23.15286 & 1996 \\
\hline & 50 & Budzisk & 53.243317 & 23.34981 & 1983 \\
\hline & 51 & Wilcza jama & 53.354164 & 23.30861 & 1983 \\
\hline & 52 & Trzcianka & 53.467007 & 23.35709 & 1983 \\
\hline & 60 & Sokołda & 53.247223 & 23.46676 & 1983 \\
\hline XU & 04 & Stobnica & 52.707057 & 16.60989 & 2003 \\
\hline
\end{tabular}


Table 2. List of the common shrew (Sorex araneus) localities.

\begin{tabular}{|c|c|c|c|c|c|}
\hline \multicolumn{2}{|c|}{ UTM square } & \multirow{2}{*}{$\frac{\text { Localities }}{\text { Dąbrowa }}$} & \multicolumn{2}{|c|}{ Geographic coordinates } & \multirow{2}{*}{$\frac{\text { Year of capture }}{1994}$} \\
\hline $\mathrm{CE}$ & 69 & & 54.092052 & 18.87601 & \\
\hline & 89 & Lubstowo & 54.131851 & 19.19815 & 1994 \\
\hline & 89 & Nogat & 54.119534 & 19.26893 & 1993 \\
\hline \multirow[t]{2}{*}{$\mathrm{CF}$} & 13 & Barwik & 54.408142 & 18.2019 & 1985 \\
\hline & 71 & Rybina & 54.29015 & 19.117 & 1994 \\
\hline DD & 19 & Czarny Bryńsk & 53.211521 & 19.7425 & 1995 \\
\hline \multirow[t]{31}{*}{$\mathrm{DE}$} & 10 & Wlecz & 53.242672 & 19.71766 & 2004 \\
\hline & 15 & Ostrów Wielki & 53.717861 & 19.72918 & 1988 \\
\hline & 16 & Kupin & 53.844166 & 19.63652 & $\begin{array}{c}1992,1993,1995 \\
1998,1999\end{array}$ \\
\hline & 19 & Kajmy & 54.088612 & 19.72195 & 1987, 1992, 1993 \\
\hline & 19 & Pasłęk & 54.066826 & 19.66181 & $\begin{array}{c}1992,1993,1995, \\
1999\end{array}$ \\
\hline & 19 & Winiary & 54.125504 & 19.71557 & 1993 \\
\hline & 29 & Bielica & 54.085045 & 19.81677 & 1987, 1992 \\
\hline & 34 & Idzbark & 53.674194 & 20.05096 & 1993,1994 \\
\hline & 35 & Nowe Siedlisko & 53.707856 & 20.04797 & 1994 \\
\hline & 35 & Szelag & 53.77 & 19.95222 & 1994 \\
\hline & 35 & Zwierzewo & 53.707584 & 20.04746 & 1994 \\
\hline & 39 & Bażyny & 54.130836 & 20.04303 & 1993 \\
\hline & 41 & Uniszewo & 53.420922 & 20.18269 & 1993 \\
\hline & 43 & Rychnowska Wola & 53.601521 & 20.12411 & 1991 \\
\hline & 43 & Swiętajny & 53.59058 & 20.23297 & $1986,1989,1990$ \\
\hline & 44 & Gugowo & 53.641482 & 20.1364 & 1990 \\
\hline & 44 & Guzowy Młyn & 53.689999 & 20.23889 & $\begin{array}{c}1985,1986,1987 \\
1988,1994\end{array}$ \\
\hline & 44 & Guzowy Piec & 53.687389 & 20.21625 & $1989,1994,1995$ \\
\hline & 44 & Ostrowin & 53.646824 & 20.10439 & 1993 \\
\hline & 45 & Biesal & 53.721687 & 20.18575 & 1987, 1988, 1989 \\
\hline & 45 & Dłużki & 53.712532 & 20.12822 & 1993,1994 \\
\hline & 45 & Lęguty & 53.753159 & 20.16362 & 1990 \\
\hline & 45 & Podlejki & 53.730518 & 20.19109 & $\begin{array}{c}1986,1989,1990 \\
1994,1995\end{array}$ \\
\hline & 45 & Śródka & 53.701881 & 20.24254 & 1986 \\
\hline & 45 & Stare Jabłonki & 53.696808 & 20.10258 & 1989,1993 \\
\hline & 45 & Worliny & 53.777642 & 20.13291 & 1991 \\
\hline & 46 & Pełnik & 53.790482 & 20.14456 & 1985 \\
\hline & 46 & Ramoty & 53.82188 & 20.08924 & 1991, 1994 \\
\hline & 44 & Elgnówko & 53.627206 & 20.21818 & 1988, 1990 \\
\hline & 53 & Jemiołowo & 53.561832 & 20.29932 & 1990 \\
\hline & 53 & Królikowo & 53.571342 & 20.25338 & 1989 \\
\hline
\end{tabular}




\begin{tabular}{|c|c|c|c|c|}
\hline 53 & Łutynowo & 53.551113 & 20.34409 & 1990,1994 \\
\hline 53 & Pawłowo & 53.532417 & 20.27352 & 1996 \\
\hline 53 & Skansen & 53.596104 & 20.29738 & 1989 \\
\hline 54 & Gębiny & 53.66423 & 20.29576 & 1989 \\
\hline 54 & Gromel & 53.685913 & 20.28203 & 1993 \\
\hline 54 & Jagielek & 53.607777 & 20.31611 & 1986,1987 \\
\hline 54 & Mańki & 53.664074 & 20.25555 & $\begin{array}{c}1986,1987,1988,1989 \\
1990,1994,1995\end{array}$ \\
\hline 54 & Miodówko & 53.645294 & 20.37587 & 1986,1991 \\
\hline 54 & Mycyny & 53.639053 & 20.267 & $\begin{array}{c}1986,1987,1989,1990, \\
1991,1994,1995,2001, \\
2004\end{array}$ \\
\hline 54 & Tolejny & 53.614437 & 20.2474 & 1988 \\
\hline 55 & Nagłady & 53.750134 & 20.27867 & 1986 \\
\hline 55 & Naterki & 53.72871 & 20.37098 & 1994,1995 \\
\hline 55 & Peglity & 53.717918 & 20.25763 & 1986 \\
\hline 55 & Szelążek & 53.770316 & 20.36565 & 1994 \\
\hline 54 & Ameryka & 53.607841 & 20.31665 & 1988 \\
\hline 62 & Dębowa Kępa & 53.460011 & 20.52982 & 1999,2000 \\
\hline 62 & Koniuszyn & 53.446304 & 20.5096 & 1998 \\
\hline 62 & Łyna & 53.439377 & 20.40929 & 2000, 2001, 2002, 2003 \\
\hline 63 & Marózek & 53.527405 & 20.42447 & 1985 \\
\hline 63 & Selwa & 53.526417 & 20.44791 & 1996 \\
\hline 63 & Swaderki & 53.550579 & 20.41717 & 1996 \\
\hline 64 & Herta & 53.694504 & 20.41766 & 1987 \\
\hline 64 & Ruś & 53.690326 & 20.49888 & 2002 \\
\hline 65 & Bartag & 53.716759 & 20.47015 & 1986 \\
\hline 66 & Wadag & 53.818676 & 20.52251 & 2003 \\
\hline 72 & Natać Wielka & 53.511272 & 20.57244 & $\begin{array}{l}1990,1998,1999,2000 \\
2001,2002,2003,2007\end{array}$ \\
\hline 72 & Natać Wielka Stara & 53.508717 & 20.57132 & 2002 \\
\hline 72 & Zimna Woda & 53.45215 & 20.6002 & 2002 \\
\hline 73 & Bałdy & 53.599087 & 20.60773 & 2000 \\
\hline 73 & Czarny Piec & 53.547199 & 20.62372 & $\begin{array}{c}1990,1995,1998,1999, \\
2000,2005,2007\end{array}$ \\
\hline 73 & Łysowo & 53.574013 & 20.6329 & 1999,2000 \\
\hline 73 & Zgniłocha & 53.563985 & 20.56234 & $\begin{array}{c}1999,2000,2002,2003, \\
2004,2005,2007\end{array}$ \\
\hline 75 & Klebark Mały & 53.766956 & 20.56206 & 2002 \\
\hline 76 & Barczewko & 53.848541 & 20.59041 & 2003, 2004 \\
\hline 82 & Głęboczek & 53.472969 & 20.8146 & 1990 \\
\hline 84 & Michałki & 53.651475 & 20.75707 & 2006 \\
\hline 84 & Miluki & 53.667682 & 20.76514 & 2006 \\
\hline 84 & Tylkowko & 53.635724 & 20.74358 & 2006 \\
\hline
\end{tabular}




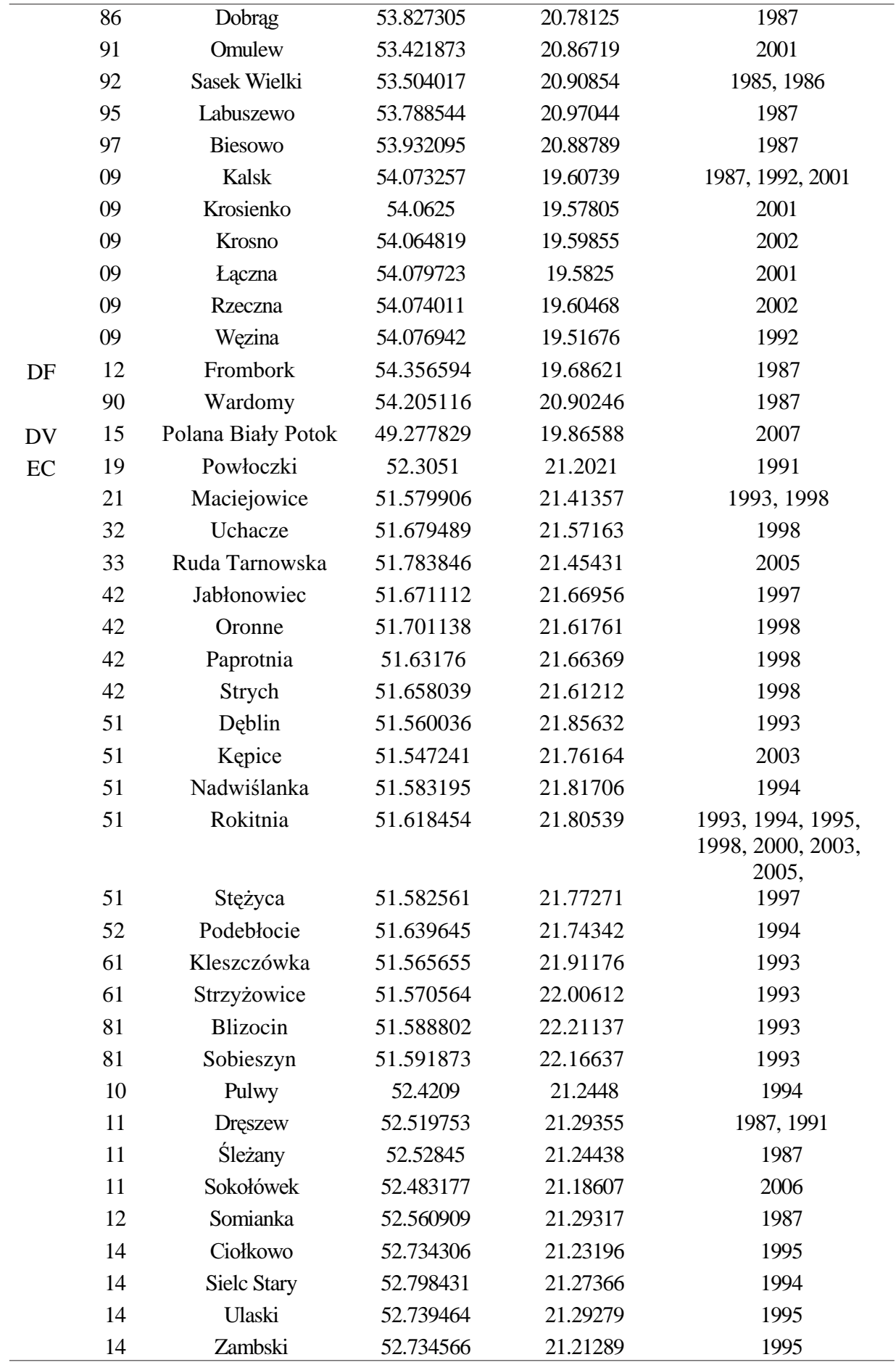




\begin{tabular}{|c|c|c|c|c|c|}
\hline \multirow[t]{25}{*}{ ED } & 10 & Pulwy & 52.4209 & 21.2448 & 1994 \\
\hline & 11 & Dręszew & 52.519753 & 21.29355 & 1987,1991 \\
\hline & 11 & Śleżany & 52.52845 & 21.24438 & 1987 \\
\hline & 11 & Sokołówek & 52.483177 & 21.18607 & 2006 \\
\hline & 12 & Somianka & 52.560909 & 21.29317 & 1987 \\
\hline & 14 & Ciołkowo & 52.734306 & 21.23196 & 1995 \\
\hline & 14 & Sielc Stary & 52.798431 & 21.27366 & 1994 \\
\hline & 14 & Ulaski & 52.739464 & 21.29279 & 1995 \\
\hline & 14 & Zambski & 52.734566 & 21.21289 & 1995 \\
\hline & 21 & Niegów & 52.508987 & 21.39112 & 1991 \\
\hline & 21 & Słopsk & 52.515728 & 21.35591 & 1991 \\
\hline & 22 & Fiszor & 52.531639 & 21.36896 & 1991 \\
\hline & 22 & Sitno & 52.59811 & 21.37628 & 1994 \\
\hline & 24 & Cygany & 52.741081 & 21.32888 & 1994 \\
\hline & 25 & Chrzczony & 52.836082 & 21.32381 & 1994 \\
\hline & 26 & Młynarze & 52.949734 & 21.40746 & 1986,1991 \\
\hline & 29 & Kierzek & 53.229378 & 21.31313 & 1985 \\
\hline & 32 & Wyszków & 52.591652 & 21.44795 & 1986 \\
\hline & 53 & Brok & 52.697723 & 21.85494 & 1986 \\
\hline & 53 & Platkownica & 52.678371 & 21.83731 & 1986 \\
\hline & 01 & Serock & 52.512646 & 21.06647 & 1986 \\
\hline & 02 & Lacha & 52.539757 & 21.09081 & 1987 \\
\hline & 03 & Popławy & 52.6989 & 21.10396 & 2005 \\
\hline & 03 & Pułtusk & 52.705456 & 21.07528 & 1986 \\
\hline & 04 & Ponikiew I & 52.725528 & 21.12161 & 2005 \\
\hline \multirow[t]{23}{*}{$\mathrm{EE}$} & 13 & Długi Borek & 53.535007 & 21.29594 & 1985 \\
\hline & 16 & Mrągowo & 53.8699 & 21.2972 & 1987 \\
\hline & 17 & Rydwagi & 53.965546 & 21.30008 & 1987 \\
\hline & 21 & Myszyniec & 53.382069 & 21.34676 & 1985 \\
\hline & 24 & Krutyń & 53.687309 & 21.43006 & 1999 \\
\hline & 33 & Karwica & 53.559994 & 21.48768 & 1986 \\
\hline & 36 & Zielony Lasek & 53.875832 & 21.5975 & 1999 \\
\hline & 39 & Doba & 54.074356 & 21.58592 & 1988 \\
\hline & 43 & Anuszewo & 53.523956 & 21.68842 & 2003 \\
\hline & 43 & Pogubie Tylne & 53.528332 & 21.74678 & 1986,2003 \\
\hline & 43 & Uściany Stare & 53.540794 & 21.68653 & 1986 \\
\hline & 43 & Wiartel & 53.601997 & 21.66701 & 2003,2004 \\
\hline & 49 & Sztynort & 54.132263 & 21.68158 & 1988 \\
\hline & 52 & Piskorzewo & 53.508083 & 21.78313 & 2003 \\
\hline & 53 & Bogumily & 53.551128 & 21.89425 & 1985 \\
\hline & 55 & Lisie Jamy & 53.710194 & 21.82203 & 1985 \\
\hline & 55 & Nowe Guty & 53.772675 & 21.85433 & 1986,1987 \\
\hline & 56 & Okartowo & 53.808395 & 21.85335 & 1986 \\
\hline & 61 & Kolno & 53.407307 & 21.92954 & 1985 \\
\hline & 79 & Czerwony Dwór & 54.130104 & 22.18694 & 1984 \\
\hline & 84 & Guty Różyńskie & 53.643765 & 22.28221 & 1985 \\
\hline & 86 & Buniaki & 53.812218 & 22.26723 & 1987 \\
\hline & 06 & Choszczewo & 53.877167 & 21.0951 & 1985 \\
\hline
\end{tabular}




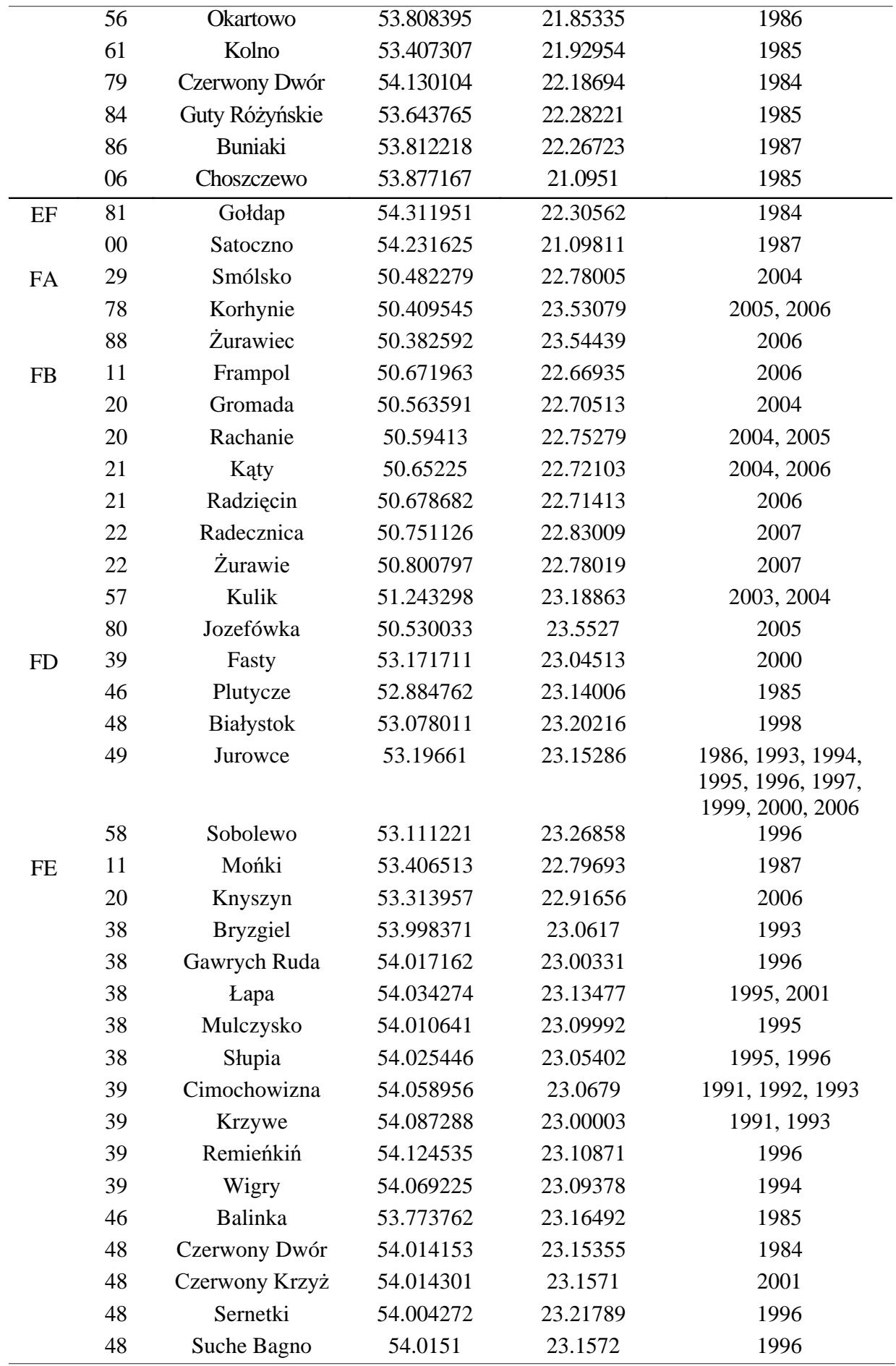




\begin{tabular}{cccccc}
\hline & 48 & Suche Bagno & 54.0151 & 23.1572 & 1996 \\
& 48 & Wysoki Most & 54.036724 & 23.20672 & 1996 \\
& 49 & Żubrówka & 54.081299 & 23.15286 & $1991,1994,1996$, \\
& 49 & Maćkowa Ruda & 54.051285 & 23.17604 & $1995,1996,2001$ \\
& 01 & Gugny & 53.348408 & 22.58955 & 1997,2000 \\
FF & 10 & Przerośl & 54.250084 & 22.65299 & 1986 \\
WV & 31 & Linowko & 53.424858 & 15.47888 & 1987 \\
XU & 04 & Piotrowo & 52.72821 & 16.49582 & 2005,2006 \\
& 04 & Stobnica & 52.707057 & 16.60989 & $2003,2005,2006$ \\
\hline
\end{tabular}

Table 3. List of the Eurasian pygmy shrew (Sorex minutus) localities.

\begin{tabular}{|c|c|c|c|c|c|}
\hline \multicolumn{2}{|c|}{ UTM square } & \multirow{2}{*}{$\frac{\text { Localities }}{\text { Dąbrowa }}$} & \multicolumn{2}{|c|}{ Geographic coordinates } & \multirow{2}{*}{$\begin{array}{c}\text { Year of capture } \\
1994\end{array}$} \\
\hline $\mathrm{CE}$ & 69 & & 54.092052 & 18.87601 & \\
\hline & 89 & Lubstowo & 54.131851 & 19.19815 & 1994 \\
\hline & 89 & Nogat & 54.119534 & 19.26893 & 1993 \\
\hline $\mathrm{CF}$ & 71 & Rybina & 54.29015 & 19.117 & 1994 \\
\hline \multirow[t]{24}{*}{ DE } & 10 & Wlecz & 53.242672 & 19.71766 & 2004 \\
\hline & 16 & Kupin & 53.844166 & 19.63652 & $\begin{array}{c}1992,1993,1995 \\
1999\end{array}$ \\
\hline & 19 & Kajmy & 54.088612 & 19.72195 & 1992, 1993 \\
\hline & 19 & Pasłęk & 54.066826 & 19.66181 & 1992, 1993, 1999 \\
\hline & 19 & Winiary & 54.125504 & 19.71557 & 1993 \\
\hline & 29 & Bielica & 54.085045 & 19.81677 & 1992 \\
\hline & 33 & Klonowo & 53.517141 & 19.9556 & 2003 \\
\hline & 34 & Idzbark & 53.674194 & 20.05096 & 1993,1994 \\
\hline & 35 & Nowe Siedlisko & 53.707856 & 20.04797 & 1994 \\
\hline & 35 & Szeląg & 53.77 & 19.95222 & 1994 \\
\hline & 35 & Zwierzewo & 53.707584 & 20.04746 & 1994 \\
\hline & 39 & Bażyny & 54.130836 & 20.04303 & 1993 \\
\hline & 41 & Uniszewo & 53.420922 & 20.18269 & 1993 \\
\hline & 43 & Rychnowska Wola & 53.601521 & 20.12411 & 1991 \\
\hline & 43 & Świętajny & 53.59058 & 20.23297 & 1989,1990 \\
\hline & 44 & Elgnówko & 53.625549 & 20.22083 & 1988,1990 \\
\hline & 44 & Guzowy Młyn & 53.689999 & 20.23889 & 1988,1994 \\
\hline & 44 & Guzowy Piec & 53.687389 & 20.21625 & 1989,1994 \\
\hline & 44 & Ostrowin & 53.646824 & 20.10439 & 1993 \\
\hline & 45 & Biesal & 53.721687 & 20.18575 & 1989 \\
\hline & 45 & Dłużki & 53.712532 & 20.12822 & 1993,1994 \\
\hline & 45 & Lęguty & 53.753159 & 20.16362 & 1990 \\
\hline & 45 & Podlejki & 53.730518 & 20.19109 & 1989, 1990, 1994 \\
\hline & 45 & Stare Jabłonki & 53.696808 & 20.10258 & 1989, 1993 \\
\hline
\end{tabular}




\begin{tabular}{|c|c|c|c|c|}
\hline 45 & Worliny & 53.779686 & 20.13292 & 1991 \\
\hline 46 & Ramoty & 53.82188 & 20.08924 & 1994 \\
\hline 53 & Jemiołowo & 53.561832 & 20.29932 & 1990 \\
\hline 53 & Królikowo & 53.571342 & 20.25338 & 1989 \\
\hline 53 & Łutynowo & 53.551113 & 20.34409 & 1990,1994 \\
\hline 53 & Pawłowo & 53.532417 & 20.27352 & 1996 \\
\hline 54 & Ameryka & 53.607841 & 20.31665 & 1988 \\
\hline 54 & Gębiny & 53.66423 & 20.29576 & 1989 \\
\hline 54 & Gromel & 53.685913 & 20.28203 & 1993 \\
\hline 54 & Mańki & 53.664074 & 20.25555 & $\begin{array}{c}1989,1990,1994,1987, \\
1988\end{array}$ \\
\hline 54 & Miodówko & 53.645294 & 20.37587 & 1991 \\
\hline 54 & Mycyny & 53.639053 & 20.267 & $\begin{array}{c}1989,1990,1991,1994, \\
1995,2001\end{array}$ \\
\hline 54 & Tolejny & 53.614437 & 20.2474 & 1988 \\
\hline 55 & Naterki & 53.72871 & 20.37098 & 1994, 1995 \\
\hline 63 & Selwa & 53.526417 & 20.44791 & 1996 \\
\hline 64 & Ruś & 53.690326 & 20.49888 & 2002 \\
\hline 62 & Dębowa Kępa & 53.460011 & 20.52982 & 2000 \\
\hline 62 & Łyna & 53.43927 & 20.40728 & 2000, 2001, 2002, 2003 \\
\hline 72 & Natać Wielka & 53.511272 & 20.57244 & $\begin{array}{c}1990,2000,2001 \\
2002,2007\end{array}$ \\
\hline 72 & Natać Wielka Stara & 53.508717 & 20.57132 & 2002 \\
\hline 72 & Zimna Woda & 53.45215 & 20.6002 & 2002 \\
\hline 73 & Bałdy & 53.599087 & 20.60773 & 2000 \\
\hline 73 & Czarny Piec & 53.547199 & 20.62372 & $\begin{array}{c}1990,1995,2000,2005, \\
2007\end{array}$ \\
\hline 73 & Zgniłocha & 53.563985 & 20.56234 & $\begin{array}{c}2002,2003,2004,2005, \\
2007\end{array}$ \\
\hline 75 & Klebark Mały & 53.766956 & 20.56206 & 2002 \\
\hline 76 & Barczewko & 53.848541 & 20.59041 & 2003,2004 \\
\hline 82 & Głęboczek & 53.472969 & 20.8146 & 1990 \\
\hline 84 & Michałki & 53.651475 & 20.75707 & 2006 \\
\hline 84 & Miluki & 53.667682 & 20.76514 & 2006 \\
\hline 84 & Tylkowko & 53.635724 & 20.74358 & 2006 \\
\hline 86 & Dobrag & 53.827305 & 20.78125 & 1987 \\
\hline 91 & Omulew & 53.421873 & 20.86719 & 2001 \\
\hline 97 & Biesowo & 53.932095 & 20.88789 & 1987 \\
\hline 09 & Kalsk & 54.073257 & 19.60739 & 1992 \\
\hline 09 & Krosienko & 54.0625 & 19.57805 & 2001 \\
\hline 09 & Krosno & 54.064819 & 19.59855 & 2002 \\
\hline 09 & Łączna & 54.079723 & 19.5825 & 2001 \\
\hline 09 & Rzeczna konie & 54.074011 & 19.60468 & 2002 \\
\hline 09 & Węzina & 54.076942 & 19.51676 & 1992 \\
\hline
\end{tabular}




\begin{tabular}{|c|c|c|c|c|c|}
\hline DV & 15 & Polana Biały Potok & 49.277829 & 19.86588 & 2007 \\
\hline \multirow[t]{16}{*}{ EC } & 19 & Powłoczki & 52.3051 & 21.2021 & 1991 \\
\hline & 21 & Maciejowice & 51.579906 & 21.41357 & 1993, 1998 \\
\hline & 32 & Uchacze & 51.679489 & 21.57163 & 1998 \\
\hline & 33 & Ruda Tarnowska & 51.783846 & 21.45431 & 2005 \\
\hline & 42 & Jabłonowiec & 51.671112 & 21.66956 & 1997 \\
\hline & 42 & Oronne & 51.701138 & 21.61761 & 1998 \\
\hline & 42 & Strych & 51.658039 & 21.61212 & 1998 \\
\hline & 51 & Dęblin & 51.560036 & 21.85632 & 1993 \\
\hline & 51 & Krukówka & 51.598503 & 21.83536 & 1997 \\
\hline & 51 & Nadwiślanka & 51.583195 & 21.81706 & 1994 \\
\hline & 51 & Rokitnia & 51.618454 & 21.80539 & $\begin{array}{c}1993,1994,1998 \\
2003,2005\end{array}$ \\
\hline & 51 & Stężyca & 51.582561 & 21.77271 & 1997 \\
\hline & 52 & Podebłocie & 51.639645 & 21.74342 & 1994 \\
\hline & 61 & Kleszczówka & 51.565655 & 21.91176 & 1993 \\
\hline & 61 & Strzyżowice & 51.570564 & 22.00612 & 1993 \\
\hline & 81 & Blizocin & 51.588802 & 22.21137 & 1993 \\
\hline \multirow[t]{15}{*}{ ED } & 10 & Pulwy & 52.4209 & 21.2448 & 1994 \\
\hline & 11 & Dręszew & 52.519753 & 21.29355 & 1991 \\
\hline & 11 & Sokołówek & 52.483307 & 21.18612 & 2006 \\
\hline & 14 & Ciołkowo & 52.734306 & 21.23196 & 1995 \\
\hline & 14 & Sielc Stary & 52.798431 & 21.27366 & 1994 \\
\hline & 14 & Ulaski & 52.739464 & 21.29279 & 1995 \\
\hline & 14 & Zambski & 52.734566 & 21.21289 & 1995 \\
\hline & 21 & Niegów & 52.508987 & 21.39112 & 1991 \\
\hline & 21 & Słopsk & 52.515728 & 21.35591 & 1991 \\
\hline & 22 & Fiszor & 52.531639 & 21.36896 & 1991 \\
\hline & 22 & Sitno & 52.59811 & 21.37628 & 1994 \\
\hline & 24 & Cygany & 52.741081 & 21.32888 & 1994 \\
\hline & 25 & Chrzczony & 52.836082 & 21.32381 & 1994 \\
\hline & 26 & Młynarze & 52.949734 & 21.40746 & 1991 \\
\hline & 03 & Popławy & 52.6989 & 21.10396 & 2005 \\
\hline \multirow[t]{4}{*}{$\mathrm{EE}$} & 43 & Wiartel & 53.59362 & 21.67953 & 2004 \\
\hline & 52 & Piskorzewo & 53.508083 & 21.78313 & 2003 \\
\hline & 53 & Pogubie & 53.558155 & 21.76566 & 2003 \\
\hline & 79 & Czerwony Dwór & 54.130104 & 22.18694 & 1984 \\
\hline $\mathrm{EF}$ & 00 & Sątoczno & 54.231625 & 21.09811 & 1987 \\
\hline \multirow[t]{3}{*}{ FA } & 29 & Smólsko & 50.482279 & 22.78005 & 2004 \\
\hline & 78 & Korhynie & 50.409545 & 23.53079 & 2005,2006 \\
\hline & 88 & Żurawce & 50.382592 & 23.54439 & 2005 \\
\hline
\end{tabular}


DISTRIBUTION OF SORICOMORPHA AND RODENTIA SPECIES IN POLAND

\begin{tabular}{|c|c|c|c|c|c|}
\hline \multirow[t]{7}{*}{ FB } & 20 & Gromada & 50.565827 & 22.70347 & 2004 \\
\hline & 20 & Rachanie & 50.59413 & 22.75279 & 2005 \\
\hline & 20 & Rapy Dylańskie & 50.59413 & 22.75279 & 2004 \\
\hline & 21 & Kąty & 50.65225 & 22.72103 & 2004,2006 \\
\hline & 21 & Radzięcin & 50.678682 & 22.71413 & 2006 \\
\hline & 22 & Radecznica & 50.751126 & 22.83009 & 2007 \\
\hline & 22 & Żurawie & 50.800797 & 22.78019 & 2007 \\
\hline \multirow[t]{9}{*}{ FD } & 39 & Fasty & 53.171711 & 23.04513 & 2000 \\
\hline & 49 & Jurowce & 53.19661 & 23.15286 & 1993, 1996, 1999 \\
\hline & 48 & Lotnisko & 53.078011 & 23.20216 & 1998 \\
\hline & 58 & Sobolewo & 53.111221 & 23.26858 & 1996 \\
\hline & 59 & Krasne & 53.196367 & 23.29969 & 1983 \\
\hline & 59 & Krasny Las & 53.173611 & 23.3375 & 1983 \\
\hline & 59 & Podsupraśl & 53.206322 & 23.33534 & 1983 \\
\hline & 68 & Żednia & 53.090096 & 23.46989 & 1983 \\
\hline & 69 & Surażkowo & 53.211678 & 23.45654 & 1983 \\
\hline \multirow[t]{22}{*}{ FE } & 38 & Bryzgiel & 53.998371 & 23.0617 & 1993 \\
\hline & 38 & Gawrych Ruda & 54.017162 & 23.00331 & 1996 \\
\hline & 38 & Mulczysko & 53.992493 & 23.10014 & 1995 \\
\hline & 39 & Cimochowizna & 54.058956 & 23.0679 & 1991, 1992, 1993 \\
\hline & 39 & Krzywe & 54.087288 & 23.00003 & 1993 \\
\hline & 39 & Remieńkiń & 54.124535 & 23.10871 & 1996 \\
\hline & 39 & Wigry & 54.069225 & 23.09378 & 1994 \\
\hline & 40 & Karczmisko & 53.299759 & 23.18604 & 1983 \\
\hline & 48 & Czerwony Krzyż & 54.014301 & 23.1571 & 2001 \\
\hline & 48 & Sernetki & 54.004272 & 23.21789 & 1996 \\
\hline & 48 & Suche Bagno & 54.0151 & 23.1572 & 1996, 2001 \\
\hline & 48 & Tobołowo & 53.967567 & 23.1464 & 2001 \\
\hline & 48 & Wysoki Most & 54.036724 & 23.20672 & 1996 \\
\hline & 48 & Zakąty & 54.014301 & 23.1571 & 1993 \\
\hline & 49 & Żubrówka & 54.081299 & 23.15286 & $\begin{array}{c}1991,1994,1996, \\
2001\end{array}$ \\
\hline & 49 & Łapa & 54.049919 & 23.15199 & 1995, 2001 \\
\hline & 49 & Maćkowa Ruda & 54.051285 & 23.17604 & 1995, 1996, 2001 \\
\hline & 50 & Budzisk & 53.243317 & 23.34981 & 1983 \\
\hline & 51 & Machnacz & 53.330753 & 23.31183 & 1983 \\
\hline & 51 & Wilcza Jama & 53.354164 & 23.30861 & 1983 \\
\hline & 52 & Trzcianka & 53.467007 & 23.35709 & 1983 \\
\hline & 60 & Sokołda & 53.247223 & 23.46676 & 1983 \\
\hline FF & 40 & Słupia & 54.138 & 23.211 & 1995,1996 \\
\hline \multirow[t]{2}{*}{ XU } & 04 & Piotrowo & 52.72821 & 16.49582 & 2005,2006 \\
\hline & 04 & Stobnica & 52.707057 & 16.60989 & $2003,2005,2006$ \\
\hline
\end{tabular}


Table 4. List of the Eurasian water shrew (Neomys fodiens) localities.

\begin{tabular}{|c|c|c|c|c|c|}
\hline \multicolumn{2}{|c|}{ UTM square } & \multirow{2}{*}{$\begin{array}{c}\text { Localities } \\
\text { Wlecz }\end{array}$} & \multicolumn{2}{|c|}{ Geographic coordinates } & \multirow{2}{*}{$\begin{array}{c}\text { Year of capture } \\
2004\end{array}$} \\
\hline $\mathrm{DE}$ & 10 & & 53.242672 & 19.71766 & \\
\hline & 16 & Kupin & 53.844166 & 19.63652 & 1992, 1993, 1999 \\
\hline & 19 & Kajmy & 54.088612 & 19.72195 & 1992,1993 \\
\hline & 19 & Pasłęk & 54.066826 & 19.66181 & 1992,1993 \\
\hline & 19 & Winiary & 54.125504 & 19.71557 & 1993 \\
\hline & 29 & Bielica & 54.085045 & 19.81677 & 1992 \\
\hline & 33 & Klonowo & 53.517141 & 19.9556 & 2003 \\
\hline & 34 & Idzbark & 53.674194 & 20.05096 & 1993 \\
\hline & 35 & Szeląg & 53.77 & 19.95222 & 1994 \\
\hline & 39 & Bażyny & 54.130836 & 20.04303 & 1993 \\
\hline & 43 & Świętajny & 53.59058 & 20.23297 & 1989,1990 \\
\hline & 44 & Elgnówko & 53.627206 & 20.21818 & 1988,1990 \\
\hline & 44 & Guzowy Młyn & 53.689999 & 20.23889 & 1988,1994 \\
\hline & 44 & Guzowy Piec & 53.687389 & 20.21625 & 1989,1994 \\
\hline & 44 & Ostrowin & 53.646824 & 20.10439 & 1993 \\
\hline & 45 & Biesal & 53.721687 & 20.18575 & 1989 \\
\hline & 45 & Łęguty & 53.753159 & 20.16362 & 1990 \\
\hline & 45 & Podlejki & 53.730518 & 20.19109 & 1989, 1990, 1994 \\
\hline & 45 & Stare Jabłonki & 53.696808 & 20.10258 & 1989 \\
\hline & 46 & Ramoty & 53.82188 & 20.08924 & 1994 \\
\hline & 53 & Królikowo & 53.571342 & 20.25338 & 1989 \\
\hline & 53 & Łutynowo & 53.551113 & 20.34409 & 1990,1994 \\
\hline & 54 & Gębiny & 53.66423 & 20.29576 & 1989 \\
\hline & 54 & Gromel & 53.685913 & 20.28203 & 1993 \\
\hline & 54 & Mańki & 53.664074 & 20.25555 & $\begin{array}{c}1987,1988,1989 \\
1990,1994\end{array}$ \\
\hline & 54 & Miodówko & 53.645294 & 20.37587 & 1991 \\
\hline & 54 & Mycyny & 53.639053 & 20.267 & $\begin{array}{c}1989,1990,1991, \\
1994,1995\end{array}$ \\
\hline & 55 & Naterki & 53.72871 & 20.37098 & 1994, 1995 \\
\hline & 62 & Łyna & 53.439377 & 20.40929 & 2000, 2001, 2002 \\
\hline & 64 & Ruś & 53.690326 & 20.49888 & 2002 \\
\hline & 72 & Natać Wielka Stara & 53.508717 & 20.57132 & 2002 \\
\hline & 72 & Zimna Woda & 53.45215 & 20.6002 & 2002 \\
\hline & 73 & Czarny Piec & 53.547199 & 20.62372 & 1990,1995 \\
\hline & 73 & Łąki Zgniłocha & 53.561932 & 20.56187 & 1990 \\
\hline & 73 & Zgniłocha & 53.563985 & 20.56234 & 2002, 2004, 2005 \\
\hline & 75 & Klebark Mały & 53.766956 & 20.56206 & 2002 \\
\hline & 76 & Barczewko & 53.848541 & 20.59041 & 2004 \\
\hline & 84 & Michałki & 53.651475 & 20.75707 & 2006 \\
\hline & 86 & Dobrąg & 53.827305 & 20.78125 & 1987 \\
\hline & 09 & Krosienko & 54.0625 & 19.57805 & 2001 \\
\hline & 09 & Kalsk & 54.073257 & 19.60739 & 1992 \\
\hline & 09 & Węzina & 54.076942 & 19.51676 & 1992 \\
\hline
\end{tabular}




\begin{tabular}{|c|c|c|c|c|c|}
\hline & 09 & Krosienko & 54.0625 & 19.57805 & 2001 \\
\hline & 09 & Kalsk & 54.073257 & 19.60739 & 1992 \\
\hline & 09 & Węzina & 54.076942 & 19.51676 & 1992 \\
\hline \multirow[t]{8}{*}{ EC } & 21 & Maciejowice & 51.579906 & 21.41357 & 1998 \\
\hline & 42 & Jabłonowiec & 51.671112 & 21.66956 & 1997 \\
\hline & 42 & Oronne & 51.701138 & 21.61761 & 1998 \\
\hline & 51 & Nadwiślanka & 51.583195 & 21.81706 & 1994 \\
\hline & 51 & Rokitnia & 51.618454 & 21.80539 & 1994, 1998, 2005 \\
\hline & 61 & Kleszczówka & 51.565655 & 21.91176 & 1993 \\
\hline & 61 & Strzyżowice & 51.570564 & 22.00612 & 1993 \\
\hline & 81 & Blizocin & 51.588802 & 22.21137 & 1993 \\
\hline \multirow[t]{5}{*}{ ED } & 11 & Dręszew & 52.519753 & 21.29355 & 1991 \\
\hline & 14 & Sielc Stary & 52.798431 & 21.27366 & 1994 \\
\hline & 14 & Zambski & 52.734566 & 21.21289 & 1995 \\
\hline & 21 & Słopsk & 52.515728 & 21.35591 & 1991 \\
\hline & 22 & Fiszor & 52.531639 & 21.36896 & 1991 \\
\hline \multirow[t]{2}{*}{ FA } & 78 & Korhynie & 50.409545 & 23.53079 & 2005 \\
\hline & 88 & Plebanka & 50.404286 & 23.66052 & 2005 \\
\hline \multirow[t]{4}{*}{ FB } & 20 & Rachanie & 50.59413 & 22.75279 & 2005 \\
\hline & 21 & Kąty & 50.65225 & 22.72103 & 2004,2006 \\
\hline & 22 & Radecznica & 50.751126 & 22.83009 & 2007 \\
\hline & 22 & Żurawie & 50.800797 & 22.78019 & 2007 \\
\hline \multirow[t]{7}{*}{ FD } & 39 & Fasty & 53.171711 & 23.04513 & 2000 \\
\hline & 49 & Jurowce & 53.19661 & 23.15286 & $1996,1999,2000$ \\
\hline & 59 & Krasne & 53.196367 & 23.29969 & 1983 \\
\hline & 59 & Krasny Las & 53.173611 & 23.3375 & 1983 \\
\hline & 59 & Podsupraśl & 53.206322 & 23.33534 & 1983 \\
\hline & 68 & Żednia & 53.090096 & 23.46989 & 1983 \\
\hline & 69 & Surażkowo & 53.211678 & 23.45654 & 1983 \\
\hline \multirow[t]{13}{*}{ FE } & 38 & Mulczysko & 53.992493 & 23.10014 & 1995 \\
\hline & 39 & Cimochowizna & 54.058956 & 23.0679 & 1991, 1992, 1993 \\
\hline & 39 & Remieńkiń & 54.124535 & 23.10871 & 1996 \\
\hline & 39 & Wigry & 54.069225 & 23.09378 & 1994 \\
\hline & 40 & Karczmisko & 53.299759 & 23.18604 & 1983 \\
\hline & 48 & Wysoki Most & 54.036724 & 23.20672 & 1996 \\
\hline & 49 & Maćkowa Ruda & 54.051285 & 23.17604 & 1996, 2001 \\
\hline & 49 & Żubrówka & 54.081299 & 23.15286 & 1991, 1994 \\
\hline & 50 & Budzisk & 53.243317 & 23.34981 & 1983 \\
\hline & 51 & Machnacz & 53.330753 & 23.31183 & 1983 \\
\hline & 51 & Wilcza Jama & 53.354164 & 23.30861 & 1983 \\
\hline & 52 & Trzcianka & 53.467007 & 23.35709 & 1983 \\
\hline & 60 & Sokołda & 53.247223 & 23.46676 & 1983 \\
\hline FF & 40 & Słupia & 54.138 & 23.211 & 1995 \\
\hline
\end{tabular}


Table 5. List of the bank vole (Myodes glareolus) localities.

\begin{tabular}{|c|c|c|c|c|c|}
\hline \multicolumn{2}{|c|}{ UTM square } & \multirow{2}{*}{$\begin{array}{l}\text { Localities } \\
\text { Dąbrowa }\end{array}$} & \multicolumn{2}{|c|}{ Geographic coordinates } & \multirow{2}{*}{$\begin{array}{c}\text { Year of capture } \\
1994\end{array}$} \\
\hline $\mathrm{CE}$ & 69 & & 54.09205 & 18.87601 & \\
\hline & 89 & Lubstowo & 54.13185 & 19.19815 & 1994 \\
\hline & 89 & Nogat & 54.11953 & 19.26893 & 1993 \\
\hline $\mathrm{CF}$ & 71 & Rybina & 54.29015 & 19.117 & 1994 \\
\hline $\mathrm{DE}$ & 10 & Wlecz & 53.24267 & 19.71766 & 2004 \\
\hline & 16 & Kupin & 53.84417 & 19.63652 & $\begin{array}{c}1992,1993,1995, \\
1999\end{array}$ \\
\hline & 19 & Kajmy & 54.08861 & 19.72195 & 1992,1993 \\
\hline & 19 & Pasłęk & 54.06683 & 19.66181 & $\begin{array}{c}1992,1993,1995 \\
1999\end{array}$ \\
\hline & 19 & Winiary & 54.1255 & 19.71557 & 1993 \\
\hline & 29 & Bielica & 54.08505 & 19.81677 & 1992 \\
\hline & 33 & Klonowo & 53.51714 & 19.9556 & 2003 \\
\hline & 34 & Idzbark & 53.67419 & 20.05096 & 1993, 1994 \\
\hline & 35 & Nowe Siedlisko & 53.70786 & 20.04797 & 1994 \\
\hline & 35 & Zwierzewo & 53.70758 & 20.04746 & 1994 \\
\hline & 39 & Bażyny & 54.13084 & 20.04303 & 1993 \\
\hline & 41 & Uniszewo & 53.42092 & 20.18269 & 1993 \\
\hline & 44 & Elgnówko & 53.62721 & 20.21818 & 1988 \\
\hline & 44 & Guzowy Młyn & 53.69 & 20.23889 & 1994 \\
\hline & 44 & Guzowy Piec & 53.68739 & 20.21625 & 1994 \\
\hline & 44 & Ostrowin & 53.64682 & 20.10439 & 1993 \\
\hline & 45 & Dłużki & 53.71253 & 20.12822 & 1994 \\
\hline & 45 & Lęguty & 53.75316 & 20.16362 & 1990 \\
\hline & 45 & Podlejki & 53.73052 & 20.19108 & 1994 \\
\hline & 45 & Stare Jabłonki & 53.69681 & 20.10258 & 1993 \\
\hline & 45 & Worliny & 53.77969 & 20.13292 & 1991 \\
\hline & 46 & Ramoty & 53.82188 & 20.08924 & 1994 \\
\hline & 53 & Jemiołowo & 53.56183 & 20.29932 & 1990 \\
\hline & 53 & Łutynowo & 53.55111 & 20.34409 & 1990,1994 \\
\hline & 53 & Pawłowo & 53.53242 & 20.27352 & 1996 \\
\hline & 54 & Gromel & 53.68591 & 20.28203 & 1993 \\
\hline & 54 & Mańki & 53.66407 & 20.25555 & 1987,1990 \\
\hline & 54 & Mycyny & 53.63905 & 20.267 & $\begin{array}{c}1990,1991,1994 \\
1995\end{array}$ \\
\hline & 54 & Tolejny & 53.61444 & 20.2474 & 1988 \\
\hline & 55 & Naterki & 53.72871 & 20.37098 & 1994,1995 \\
\hline & 62 & Dębowa Kępa & 53.46001 & 20.52982 & 2000 \\
\hline & 64 & Ruś & 53.69033 & 20.49888 & 2002 \\
\hline & 72 & Natać Wielka & 53.51127 & 20.57244 & 2001 \\
\hline & 72 & Natać Wielka Stara & 53.50872 & 20.57132 & 2002 \\
\hline & 73 & Czarny Piec & 53.547 & 20.62437 & 2005 \\
\hline & 73 & Zgniłocha & 53.56399 & 20.56234 & $2002,2004,2005$ \\
\hline & 76 & Barczewko & 53.84854 & 20.59041 & 2003, 2004 \\
\hline & 82 & Głęboczek & 53.47297 & 20.8146 & 1990 \\
\hline & 91 & Omulew & 53.42187 & 20.86719 & 2001 \\
\hline & 97 & Biesowo & 53.9321 & 20.88789 & 1987 \\
\hline
\end{tabular}




\begin{tabular}{|c|c|c|c|c|c|}
\hline & 97 & Biesowo & 53.9321 & 20.88789 & 1987 \\
\hline & 09 & Rzeczna & 54.07401 & 19.60468 & 2002 \\
\hline & 09 & Kalsk & 54.07326 & 19.60739 & 1992,2001 \\
\hline & 09 & Krosno & 54.06482 & 19.59855 & 2002 \\
\hline & 09 & Węzina & 54.07694 & 19.51676 & 1992 \\
\hline DF & 90 & Wardomy & 54.20512 & 20.90246 & 1987 \\
\hline \multirow[t]{16}{*}{$\mathrm{EC}$} & 19 & Powłoczki & 52.3051 & 21.2021 & 1991 \\
\hline & 21 & Maciejowice & 51.57991 & 21.41357 & 1993, 1998 \\
\hline & 33 & Ruda Tarnowska & 51.78385 & 21.45431 & 2005 \\
\hline & 42 & Jabłonowiec & 51.67111 & 21.66956 & 1997 \\
\hline & 42 & Strych & 51.65804 & 21.61212 & 1998 \\
\hline & 51 & Dęblin & 51.56004 & 21.85632 & 1993 \\
\hline & 51 & Kępica & 51.54737 & 21.76181 & 2003 \\
\hline & 51 & Krukówka & 51.5985 & 21.83536 & 1997 \\
\hline & 51 & Nadwiślanka & 51.5832 & 21.81706 & 1994 \\
\hline & 51 & Rokitnia & 51.61845 & 21.80539 & $\begin{array}{c}1993,1994,1998 \\
2003,2005\end{array}$ \\
\hline & 51 & Stężyca & 51.58256 & 21.77271 & 1997 \\
\hline & 52 & Podebłocie & 51.63965 & 21.74342 & 1994 \\
\hline & 61 & Kleszczówka & 51.56566 & 21.91176 & 1993 \\
\hline & 61 & Strzyżowice & 51.57056 & 22.00612 & 1993 \\
\hline & 81 & Blizocin & 51.5888 & 22.21137 & 1993 \\
\hline & 81 & Sobieszyn & 51.59187 & 22.16637 & 1993 \\
\hline \multirow[t]{13}{*}{ ED } & 10 & Pulwy & 52.4209 & 21.2448 & 1994 \\
\hline & 11 & Dręszew & 52.51975 & 21.29355 & 1991 \\
\hline & 14 & Ciołkowo & 52.73431 & 21.23196 & 1995 \\
\hline & 14 & Sielc Stary & 52.79843 & 21.27366 & 1994 \\
\hline & 14 & Ulaski & 52.73946 & 21.29279 & 1995 \\
\hline & 14 & Zambski & 52.73457 & 21.21289 & 1995 \\
\hline & 21 & Niegów & 52.50899 & 21.39112 & 1991 \\
\hline & 21 & Słopsk & 52.51573 & 21.35591 & 1991 \\
\hline & 22 & Fiszor & 52.53164 & 21.36896 & 1991 \\
\hline & 24 & Cygany & 52.74108 & 21.32888 & 1994 \\
\hline & 25 & Chrzczony & 52.83608 & 21.32381 & 1994 \\
\hline & 03 & Popławy & 52.6989 & 21.10396 & 2005 \\
\hline & 04 & Ponikiew & 52.72553 & 21.12161 & 2005 \\
\hline $\mathrm{EE}$ & 79 & Czerwony Dwór & 54.1301 & 22.18694 & 1984 \\
\hline $\mathrm{EF}$ & 00 & Sątoczno & 54.23163 & 21.09811 & 1987 \\
\hline \multirow[t]{3}{*}{ FA } & 29 & Smólsko & 50.48228 & 22.78005 & 2004 \\
\hline & 88 & Plebanka & 50.40429 & 23.66052 & 2005 \\
\hline & 88 & Żurawce & 50.38259 & 23.54439 & 2005 \\
\hline \multirow[t]{2}{*}{ FB } & 20 & Gromada & 50.56583 & 22.70347 & 2004 \\
\hline & 21 & Kąty & 50.65225 & 22.72103 & 2004 \\
\hline \multirow[t]{5}{*}{ FD } & 48 & Białystok & 53.07801 & 23.20216 & 1998 \\
\hline & 49 & Jurowce & 53.19661 & 23.15286 & $\begin{array}{c}1993,1996,2000, \\
2006\end{array}$ \\
\hline & 58 & Sobolewo & 53.11122 & 23.26858 & 1996 \\
\hline & 59 & Krasne & 53.19637 & 23.29969 & 1983 \\
\hline & 59 & Krasny Las & 53.17361 & 23.3375 & 1983 \\
\hline
\end{tabular}




\begin{tabular}{|c|c|c|c|c|c|}
\hline & 59 & Podsupraśl & 53.20632 & 23.33534 & 1983 \\
\hline & 68 & Żednia & 53.0901 & 23.46989 & 1983 \\
\hline & 69 & Surażkowo & 53.21168 & 23.45654 & 1983 \\
\hline \multirow[t]{20}{*}{$\mathrm{FE}$} & 21 & Knyszyn & 53.32651 & 22.92031 & 2006 \\
\hline & 38 & Bryzgiel & 53.99837 & 23.0617 & 1993 \\
\hline & 38 & Gawrych Ruda & 54.01716 & 23.00331 & 1996 \\
\hline & 38 & Mulczysko & 53.99249 & 23.10014 & 1995 \\
\hline & 39 & Cimochowizna & 54.05896 & 23.0679 & 1991, 1992, 1993 \\
\hline & 39 & Krzywe & 54.08729 & 23.00003 & 1993 \\
\hline & 39 & Remieńkiń & 54.12454 & 23.10871 & 1996 \\
\hline & 40 & Karczmisko & 53.29976 & 23.18604 & 1983 \\
\hline & 48 & Czerwony Krzyż & 54.0143 & 23.1571 & 1993, 2001 \\
\hline & 48 & Suche Bagno & 54.0151 & 23.1572 & 1996 \\
\hline & 48 & Wysoki Most & 54.03672 & 23.20672 & 1996 \\
\hline & 49 & Żubrówka & 54.0813 & 23.15286 & 1991, 1994, 2001 \\
\hline & 49 & Łapa & 54.04992 & 23.15199 & 1995, 2001 \\
\hline & 49 & Maćkowa Ruda & 54.05129 & 23.17604 & 1995, 1996, 2001 \\
\hline & 50 & Budzisk & 53.24332 & 23.34981 & 1983 \\
\hline & 51 & Machnacz & 53.33075 & 23.31183 & 1983 \\
\hline & 51 & Wilcza Jama & 53.35416 & 23.30861 & 1983 \\
\hline & 52 & Trzcianka & 53.46701 & 23.35709 & 1983 \\
\hline & 60 & Sokołda & 53.24722 & 23.46676 & 1983 \\
\hline & 48 & Sernetki & 54.00427 & 23.21789 & 1996 \\
\hline \multirow[t]{3}{*}{ FF } & 21 & Udziejek & 54.26051 & 22.87174 & 2001 \\
\hline & 21 & Wodziłki & 54.23839 & 22.84168 & 2000 \\
\hline & 40 & Słupia & 54.138 & 23.211 & 1995,1996 \\
\hline \multirow[t]{2}{*}{ XU } & 04 & Piotrowo & 52.72821 & 16.49582 & 2005 \\
\hline & 04 & Stobnica & 52.70706 & 16.60989 & 2003, 2005, 2006 \\
\hline
\end{tabular}

Table 6. List of the water vole (Arvicola terrestris) localities.

\begin{tabular}{|c|c|c|c|c|c|}
\hline \multicolumn{2}{|c|}{ UTM square } & \multirow{2}{*}{$\begin{array}{c}\text { Localities } \\
\text { Dłużki }\end{array}$} & \multicolumn{2}{|c|}{ Geographic coordinates } & \multirow{2}{*}{$\begin{array}{c}\text { Year of capture } \\
1993\end{array}$} \\
\hline $\mathrm{DE}$ & 45 & & 53,71253 & 20,12822 & \\
\hline & 54 & Gębiny & 53,66423 & 20,29576 & 1989 \\
\hline & 54 & Mańki & 53,66604 & 20,25569 & 1987,1988 \\
\hline & 54 & Mycyny & 53,63905 & 20,267 & 1994 \\
\hline & 44 & Ostrowin & 53,64682 & 20,10439 & 1993 \\
\hline & 19 & Pasłęk & 54,06683 & 19,66181 & 1993 \\
\hline & 41 & Uniszewo & 53,42092 & 20,18269 & 1993 \\
\hline FE & 49 & Żubrówka & 54,0813 & 23,15286 & 1994 \\
\hline
\end{tabular}

Table 7. List of the European pine vole (Pitymys subterraneus) localities.

\begin{tabular}{cccccc}
\hline \multicolumn{2}{c}{ UTM square } & Localities & \multicolumn{2}{c}{ Geographic coordinates } & Year of capture \\
\hline FD & 69 & Surażkowo & 53.21168 & 23.45654 & 1983 \\
FE & 51 & Machnacz & 53.33075 & 23.31183 & 1983 \\
& 52 & Trzcianka & 53.46701 & 23.35709 & 1983 \\
\hline
\end{tabular}


Table 8. List of the field vole (Microtus agrestis) localities.

\begin{tabular}{cccccc}
\hline \multicolumn{2}{c}{ UTM square } & Localities & \multicolumn{2}{c}{ Geographic coordinates } & Year of capture \\
\hline DE & 44 & Guzowy Młyn & 53.69 & 20.23889 & 1994 \\
& 44 & Guzowy Piec & 53.68739 & 20.21625 & 1994 \\
& 53 & Lutynowo & 53.55111 & 20.34409 & 1994 \\
& 53 & Pawłowo & 53.53242 & 20.27352 & 1996 \\
& 54 & Mycyny & 53.63905 & 20.267 & 1994 \\
& 62 & Lyna & 53.43938 & 20.40929 & 2001 \\
& 72 & Natać Wielka & 53.50724 & 20.56848 & 2000 \\
& 73 & Bałdy & 53.59909 & 20.60773 & 2000 \\
& 73 & Zgniłocha & 53.56399 & 20.56234 & 2004 \\
DE & 15 & Polana Biały Potok & 49.27783 & 19.86588 & 2007 \\
FA & 79 & Czerwony Dwór & 54.1301 & 22.18694 & 1984 \\
FD & 88 & Żurawce & 50.38259 & 23.54439 & 2005 \\
& 59 & Krasne & 53.19637 & 23.29969 & 1983 \\
& 59 & Krasny Las & 53.17361 & 23.3375 & 1983 \\
& 68 & Żednia & 53.0901 & 23.46989 & 1983 \\
& 69 & Surażkowo & 53.21168 & 23.45654 & 1983 \\
FE & 40 & Karczmisko & 53.29976 & 23.18604 & 1983 \\
& 50 & Budzisk & 53.24332 & 23.34981 & 1983 \\
& 51 & Machnacz & 53.33075 & 23.31183 & 1983 \\
& 51 & Wilcza Jama & 53.35416 & 23.30861 & 1983 \\
& 52 & Trzcianka & 53.46701 & 23.35709 & 1983 \\
& 60 & Sokołda & 53.24722 & 23.46676 & 1983 \\
\hline
\end{tabular}

Table 9. List of the common vole (Microtus arvalis) localities.

\begin{tabular}{|c|c|c|c|c|c|}
\hline \multicolumn{2}{|c|}{ UTM square } & \multirow{2}{*}{$\begin{array}{c}\text { Localities } \\
\text { Kupin }\end{array}$} & \multicolumn{2}{|c|}{ Geographic coordinates } & \multirow{2}{*}{$\begin{array}{c}\text { Year of capture } \\
1999\end{array}$} \\
\hline $\mathrm{DE}$ & 16 & & 53.844166 & 19.63652 & \\
\hline & 44 & Guzowy Młyn & 53.689999 & 20.23889 & 1994 \\
\hline & 44 & Guzowy Piec & 53.687389 & 20.21625 & 1994 \\
\hline & 46 & Ramoty & 53.82188 & 20.08924 & 1994 \\
\hline & 53 & Łutynowo & 53.551113 & 20.34409 & 1994 \\
\hline & 53 & Pawłowo & 53.532417 & 20.27352 & 1996 \\
\hline & 54 & Ameryka & 53.607841 & 20.31665 & 1988 \\
\hline & 54 & Mycyny & 53.639053 & 20.267 & 1994 \\
\hline & 73 & Zgniłocha & 53.563985 & 20.56234 & 2004 \\
\hline $\mathrm{EC}$ & 51 & Rokitnia & 51.618454 & 21.80539 & 1998 \\
\hline $\mathrm{EE}$ & 79 & Czerwony Dwór & 54.130104 & 22.18694 & 1984 \\
\hline FA & 29 & Smólsko & 50.482279 & 22.78005 & 2004 \\
\hline \multirow[t]{3}{*}{ FB } & 20 & Gromada & 50.565827 & 22.70347 & 2004 \\
\hline & 20 & Rapy Dylańskie & 50.59413 & 22.75279 & 2004 \\
\hline & 21 & Kąty & 50.65225 & 22.72103 & 2004 \\
\hline
\end{tabular}




\begin{tabular}{cccccc}
\hline FD & 49 & Jurowce & 53.19661 & 23.15286 & 1996 \\
& 59 & Krasny Las & 53.173611 & 23.3375 & 1983 \\
& 68 & Żednia & 53.090096 & 23.46989 & 1983 \\
FE & 39 & Cimochowizna & 54.058956 & 23.0679 & 1991,1992 \\
& 40 & Karczmisko & 53.299759 & 23.18604 & 1983 \\
& 50 & Budzisk & 53.243317 & 23.34981 & 1983 \\
& 51 & Machnacz & 53.330753 & 23.31183 & 1983 \\
& 52 & Trzcianka & 53.467007 & 23.35709 & 1983 \\
& 60 & Sokołda & 53.247223 & 23.46676 & 1983 \\
FF & 10 & Turtul & 54.21986 & 22.80434 & 2000 \\
& 21 & Udziejek & 54.260513 & 22.87174 & 2001 \\
\hline
\end{tabular}

Table 10. List of the root vole (Microtus oeconomus) localities.

\begin{tabular}{|c|c|c|c|c|c|}
\hline \multicolumn{2}{|c|}{ UTM square } & \multirow{2}{*}{$\begin{array}{l}\text { Localities } \\
\text { Uniszewo }\end{array}$} & \multicolumn{2}{|c|}{ Geographic coordinates } & \multirow{2}{*}{$\begin{array}{c}\text { Year of capture } \\
1993\end{array}$} \\
\hline $\mathrm{DE}$ & 41 & & 53.42092 & 20.18269 & \\
\hline & 44 & Guzowy Młyn & 53.69 & 20.23889 & 1994 \\
\hline & 44 & Guzowy Piec & 53.68739 & 20.21625 & 1994 \\
\hline & 45 & Dłużki & 53.71253 & 20.12822 & 1993 \\
\hline & 45 & Podlejki & 53.73052 & 20.18109 & 1994 \\
\hline & 46 & Ramoty & 53.82188 & 20.08924 & 1994 \\
\hline & 53 & Łutynowo & 53.55111 & 20.34409 & 1990, 1994 \\
\hline & 53 & Pawłowo & 53.53242 & 20.27352 & 1996 \\
\hline & 54 & Mańki & 53.66604 & 20.25569 & 1987 \\
\hline & 54 & Mycyny & 53.63905 & 20.267 & 1994,1995 \\
\hline & 62 & Łyna & 53.43938 & 20.40929 & 2000, 2001, 2002 \\
\hline & 63 & Swaderki & 53.55058 & 20.41717 & 1996 \\
\hline & 72 & Natać Wielka & 53.51127 & 20.57244 & 2001 \\
\hline & 73 & Bałdy & 53.59909 & 20.60773 & 2000 \\
\hline & 09 & Kalsk & 54.07326 & 19.60739 & 1992 \\
\hline \multirow[t]{3}{*}{$\mathrm{EC}$} & 42 & Jabłonowiec & 51.67111 & 21.66956 & 1997 \\
\hline & 51 & Rokitnia & 51.61845 & 21.80539 & 1998 \\
\hline & 81 & Blizocin & 51.5888 & 22.21137 & 1993 \\
\hline $\mathrm{EE}$ & 79 & Czerwony Dwór & 54.1301 & 22.18694 & 1984 \\
\hline \multirow[t]{5}{*}{ FD } & 49 & Jurowce & 53.19661 & 23.15286 & 1996 \\
\hline & 59 & Krasne & 53.19637 & 23.29969 & 1983 \\
\hline & 59 & Krasny Las & 53.17361 & 23.3375 & 1983 \\
\hline & 68 & Żednia & 53.0901 & 23.46989 & 1983 \\
\hline & 69 & Surażkowo & 53.21168 & 23.45654 & 1983 \\
\hline \multirow[t]{4}{*}{$\mathrm{FE}$} & 39 & Cimochowizna & 54.05896 & 23.0679 & 1991, 1992, 1993 \\
\hline & 39 & Krzywe & 54.08729 & 23.00003 & 1993 \\
\hline & 40 & Karczmisko & 53.29976 & 23.18604 & 1983 \\
\hline & 48 & Zakąty & 54.0143 & 23.1571 & 1993 \\
\hline
\end{tabular}




\begin{tabular}{cccccc}
\hline & 50 & Budzisk & 53.24332 & 23.34981 & 1983 \\
& 51 & Machnacz & 53.33075 & 23.31183 & 1983 \\
& 51 & Wilcza Jama & 53.35416 & 23.30861 & 1983 \\
& 52 & Trzcianka & 53.46701 & 23.35709 & 1983 \\
& 60 & Sokołda & 53.24722 & 23.46676 & 1983 \\
FF & 10 & Turtul & 54.21986 & 22.80434 & 2000,2002 \\
& 21 & Wodziłki & 54.23839 & 22.84168 & 2000 \\
\hline
\end{tabular}

Table 11. List of the house mouse (Mus musculus) localities.

\begin{tabular}{cccccc}
\hline \multicolumn{2}{c}{ UTM square } & Localities & \multicolumn{2}{c}{ Geographic coordinates } & Year of capture \\
\hline DE & 16 & Kupin & 53.84417 & 19.63652 & 1992,1993 \\
& 29 & Bielica & 54.08505 & 19.81677 & 1992 \\
EC & 21 & Maciejowice & 51.57991 & 21.41357 & 1998 \\
FA & 78 & Chałupa & 50.40955 & 23.53079 & 2006 \\
& 88 & Jarczów & 50.42498 & 23.58807 & 2006 \\
& 88 & Plebanka & 50.40429 & 23.66052 & 2005 \\
& 88 & Żurawce & 50.38259 & 23.54439 & 2005 \\
FE & 38 & Bryzgiel & 53.99837 & 23.0617 & 1993 \\
& 39 & Cimochowizna & 54.05896 & 23.0679 & 1991 \\
& 51 & Wilcza Jama & 53.35416 & 23.30861 & 1983 \\
& 52 & Trzcianka & 53.46701 & 23.35709 & 1983 \\
& 60 & Sokołda & 53.24722 & 23.46676 & 1983 \\
FF & 10 & Turtul & 54.21986 & 22.80434 & 2000 \\
XU & 04 & Stobnica & 52.70706 & 16.60989 & 2006 \\
\hline
\end{tabular}

Table 12. List of the common rat (Rattus norvegicus) localities.

\begin{tabular}{cccccc}
\hline \multicolumn{2}{c}{ UTM square } & Localities & \multicolumn{2}{c}{ Geographic coordinates } & Year of capture \\
\hline \multirow{2}{*}{ FB } & 36 & Fajsławice & 51.09311 & 22.96133 & 2006 \\
& 24 & Pilaszkowice & 50.98402 & 22.84069 & 2006,2010 \\
\multirow{2}{*}{ FE } & 51 & Wilcza Jama & 53.35416 & 23.30861 & 1983 \\
& 52 & Trzcianka & 53.46701 & 23.35709 & 1983 \\
\hline
\end{tabular}

Table 13. List of the harvest mouse (Micromys minutus) localities.

\begin{tabular}{cccccc}
\hline \multicolumn{2}{c}{ UTM square } & Localities & \multicolumn{2}{c}{ Geographic coordinates } & Year of capture \\
\hline DE & 16 & Kupin & 53.84417 & 19.636515 & 1992 \\
& 19 & Kajmy & 54.08861 & 19.721945 & 1992 \\
& 19 & Pasłęk & 54.06683 & 19.661812 & 1992 \\
& Idzbark & 53.67419 & 20.050957 & 1993 \\
& 44 & Gębiny & 53.66423 & 20.295763 & 1989 \\
& Guzowy Młyn & 53.69 & 20.238888 & 1994 \\
\hline
\end{tabular}




\begin{tabular}{|c|c|c|c|c|c|}
\hline \multirow[t]{20}{*}{ 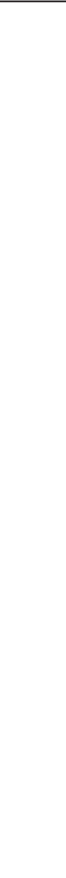 } & 44 & Guzowy Piec & 53.68739 & 20.216253 & 1994 \\
\hline & 44 & Ostrowin & 53.64682 & 20.104393 & 1993 \\
\hline & 45 & Biesal & 53.72169 & 20.185753 & 1989 \\
\hline & 45 & Łęguty & 53.75316 & 20.163618 & 1990 \\
\hline & 45 & Podlejki & 53.73052 & 2019108 & 1989,1994 \\
\hline & 46 & Ramoty & 53.82188 & 20.089239 & 1994 \\
\hline & 53 & Królikowo & 53.57134 & 20.253378 & 1989 \\
\hline & 53 & Łutynowo & 53.55111 & 20.344088 & 1994 \\
\hline & 54 & Gromel & 53.68591 & 20.282028 & 1993 \\
\hline & 54 & Mańki & 53.66407 & 20.25555 & 1989,1994 \\
\hline & 54 & Miodówko & 53.64529 & 20.375872 & 1991 \\
\hline & 54 & Mycyny & 53.63905 & 20.266998 & 1991, 1994, 1995 \\
\hline & 55 & Naterki & 53.72871 & 20.370975 & 1994 \\
\hline & 62 & Łyna & 53.43938 & 20.409292 & 2001 \\
\hline & 72 & Natać Wielka & 53.51127 & 20.572437 & 2001 \\
\hline & 73 & Zgniłocha & 53.56399 & 20.562341 & 2004 \\
\hline & 76 & Barczewko & 53.84854 & 20.59041 & 2004 \\
\hline & 84 & Michałki & 53.65148 & 20.757065 & 2006 \\
\hline & 09 & Kalsk & 54.07326 & 19.607391 & 1992 \\
\hline & 09 & Węzina & 54.07694 & 19.516756 & 1992 \\
\hline \multirow[t]{4}{*}{ EC } & 21 & Maciejowice & 51.57991 & 21.413569 & 1993 \\
\hline & 51 & Rokitnia & 51.61845 & 21.80539 & 1994 \\
\hline & 81 & Blizocin & 51.5888 & 22.21137 & 1993 \\
\hline & 81 & Sobieszyn & 51.59187 & 22.166374 & 1993 \\
\hline \multirow[t]{3}{*}{ ED } & 11 & Dręszew & 52.51975 & 21.293547 & 1991 \\
\hline & 14 & Sielc Stary & 52.79843 & 21.273661 & 1994 \\
\hline & 21 & Słopsk & 52.51573 & 21.355907 & 1991 \\
\hline \multirow[t]{3}{*}{ FA } & 29 & Smólsko & 50.48228 & 22.780048 & 2004 \\
\hline & 88 & Plebanka & 50.40429 & 23.660519 & 2005 \\
\hline & 88 & Żurawce & 50.38259 & 23.54439 & 2005 \\
\hline \multirow[t]{7}{*}{ FB } & 20 & Gromada & 50.56583 & 22.703472 & 2004 \\
\hline & 20 & Krukówka & 51.5985 & 21.835361 & 1997 \\
\hline & 20 & Rapy Dylańskie & 50.59413 & 22.752787 & 2004 \\
\hline & 21 & Kąty & 50.65225 & 22.721029 & 2004, 2006 \\
\hline & 21 & Radzięcin & 50.67868 & 22.714128 & 2006 \\
\hline & 22 & Radecznica & 50.75113 & 22.83009 & 2007 \\
\hline & 22 & Żurawie & 50.8008 & 22.78019 & 2007 \\
\hline \multirow[t]{2}{*}{ FD } & 39 & Fasty & 53.17171 & 23.045132 & 2000 \\
\hline & 49 & Jurowce & 53.19661 & 23.152861 & 1996, 1999 \\
\hline \multirow[t]{3}{*}{$\mathrm{FE}$} & 38 & Mulczysko & 53.99249 & 23.100143 & 1995 \\
\hline & 39 & Cimochowizna & 54.05896 & 23.0679 & 1991, 1992, 1993 \\
\hline & 39 & Wigry & 54.06923 & 23.093784 & 1994 \\
\hline
\end{tabular}




\begin{tabular}{ccccc}
\hline 40 & Karczmisko & 53.29976 & 23.186043 & 1983 \\
49 & Żubrówka & 54.0813 & 23.152861 & 1994 \\
49 & Łapa & 54.04992 & 23.151985 & 1995 \\
49 & Maćkowa Ruda & 54.05129 & 23.176041 & 1995 \\
51 & Wilcza Jama & 53.35416 & 23.308611 & 1983 \\
52 & Trzcianka & 53.46701 & 23.357092 & 1983 \\
\hline
\end{tabular}

Table 14. List of the yellow-necked mouse (Apodemus flavicollis) localities.

\begin{tabular}{|c|c|c|c|c|c|}
\hline \multicolumn{2}{|c|}{ UTM square } & \multirow{2}{*}{$\begin{array}{l}\text { Localities } \\
\text { Elgnówko }\end{array}$} & \multicolumn{2}{|c|}{ Geographic coordinates } & \multirow{2}{*}{$\begin{array}{c}\text { Year of capture } \\
1988\end{array}$} \\
\hline $\mathrm{DE}$ & 44 & & 53.627206 & 20.218182 & \\
\hline & 44 & Guzowy Młyn & 53.689999 & 20.238888 & 1994 \\
\hline & 44 & Guzowy Piec & 53.687389 & 20.216253 & 1994 \\
\hline & 46 & Ramoty & 53.82188 & 20.089239 & 1994 \\
\hline & 53 & Łutynowo & 53.551113 & 20.344088 & 1994 \\
\hline & 53 & Pawłowo & 53.532417 & 20.27352 & 1996 \\
\hline & 54 & Mycyny & 53.639053 & 20.266998 & 1994 \\
\hline & 64 & Ruś & 53.690326 & 20.498875 & 2002 \\
\hline DV & 15 & Polana Biały Potok & 49.277829 & 19.865878 & 2007 \\
\hline $\mathrm{EE}$ & 79 & Czerwony Dwór & 54.130104 & 22.186937 & 1984 \\
\hline \multirow[t]{2}{*}{ FA } & 78 & Korhynie & 50.40955 & 23.53079 & 2006 \\
\hline & 88 & Żurawce & 50.382592 & 23.54439 & 2005 \\
\hline \multirow[t]{6}{*}{ FD } & 48 & Lotnisko Białystok & 53.078011 & 23.202156 & 2002 \\
\hline & 59 & Krasne & 53.196367 & 23.299685 & 1983 \\
\hline & 59 & Krasny Las & 53.173611 & 23.3375 & 1983 \\
\hline & 59 & Podsupraśl & 53.206322 & 23.335337 & 1983 \\
\hline & 68 & Żednia & 53.090096 & 23.469893 & 1997 \\
\hline & 69 & Surażkowo & 53.211678 & 23.456541 & 1983 \\
\hline \multirow[t]{10}{*}{$\mathrm{FE}$} & 38 & Bryzgiel & 53.998371 & 23.061699 & 1993 \\
\hline & 39 & Cimochowizna & 54.058956 & 23.0679 & 1991,1992 \\
\hline & 39 & Krzywe & 54.087288 & 23.000028 & 1993 \\
\hline & 40 & Karczmisko & 53.299759 & 23.186043 & 1983 \\
\hline & 41 & Przewalanka & 53.325001 & 23.123055 & 1997 \\
\hline & 48 & Zakąty & 54.014301 & 23.157095 & 1993 \\
\hline & 50 & Budzisk & 53.243317 & 23.34981 & 1983 \\
\hline & 51 & Machnacz & 53.330753 & 23.311829 & 1983 \\
\hline & 51 & Wilcza Jama & 53.354164 & 23.308611 & 1983 \\
\hline & 52 & Trzcianka & 53.467007 & 23.357092 & 1983 \\
\hline \multirow[t]{3}{*}{$\mathrm{FF}$} & 10 & Turtul & 54.21986 & 22.804338 & 2000 \\
\hline & 21 & Udziejek & 54.260513 & 22.871738 & 2000 \\
\hline & 21 & Wodziłki & 54.238392 & 22.841677 & 2000 \\
\hline $\mathrm{XU}$ & 04 & Stobnica & 52.707057 & 16.609894 & 2003,2006 \\
\hline
\end{tabular}


Table 15. List of the wood mouse (Apodemus sylvaticus) localities.

\begin{tabular}{cccccc}
\hline \multicolumn{2}{l}{ UTM square } & Localities & \multicolumn{2}{c}{ Geographic coordinates } & Year of capture \\
\hline FD & 48 & Lotnisko Białystok & 53.07801 & 23.20216 & 2002 \\
FF & 21 & Udziejek & 54.26051 & 22.87174 & 2000 \\
& 21 & Wodziłki & 54.23839 & 22.84168 & 2000 \\
& 10 & Turtul & 54.21986 & 22.80434 & 2000 \\
\hline
\end{tabular}

Table 16. List of the striped field mouse (Apodemus agrarius) localities.

\begin{tabular}{|c|c|c|c|c|c|}
\hline \multicolumn{2}{|c|}{ UTM square } & \multirow{2}{*}{$\begin{array}{c}\text { Localities } \\
\text { Kupin }\end{array}$} & \multicolumn{2}{|c|}{ Geographic coordinates } & \multirow{2}{*}{$\frac{\text { Year of capture }}{1992}$} \\
\hline $\mathrm{DE}$ & 16 & & 53.84417 & 19.63652 & \\
\hline & 19 & Pasłęk & 54.06683 & 19.66181 & 1992,1993 \\
\hline & 44 & Elgnówko & 53.62721 & 20.21818 & 1988 \\
\hline & 44 & Guzowy Młyn & 53.69 & 20.23889 & 1994 \\
\hline & 44 & Guzowy Piec & 53.68739 & 20.21625 & 1994 \\
\hline & 45 & Podlejki & 53.73052 & 20191075 & 1989,1994 \\
\hline & 46 & Ramoty & 53.82188 & 20.08924 & 1994 \\
\hline & 54 & Mycyny & 53.63905 & 20.267 & 1995 \\
\hline & 62 & Łyna & 53.43927 & 20.40728 & 2003 \\
\hline & 64 & Ruś & 53.69033 & 20.49888 & 2002 \\
\hline & 72 & Natać Wielka Stara & 53.50872 & 20.57132 & 2002 \\
\hline & 75 & Klebark Mały & 53.76696 & 20.56206 & 2002 \\
\hline & 76 & Barczewko & 53.84854 & 20.59041 & 2004 \\
\hline DV & 15 & Polana Biały Potok & 49.27783 & 19.86588 & 2007 \\
\hline \multirow[t]{8}{*}{$\mathrm{EC}$} & 21 & Maciejowice & 51.57991 & 21.41357 & 1993 \\
\hline & 33 & Ruda Tarnowska & 51.78385 & 21.45431 & 2005 \\
\hline & 51 & Kępica & 51.54737 & 21.76181 & 2003 \\
\hline & 51 & Krukówka & 51.5985 & 21.83536 & 1997 \\
\hline & 51 & Rokitnia & 51.61212 & 21.79405 & 2005 \\
\hline & 61 & Kleszczówka & 51.56566 & 21.91176 & 1993 \\
\hline & 61 & Strzyżowice & 51.57056 & 22.00612 & 1993 \\
\hline & 81 & Blizocin & 51.5888 & 22.21137 & 1993 \\
\hline ED & 11 & Sokołówek & 52.48331 & 21.18612 & 2006 \\
\hline \multirow[t]{3}{*}{ FA } & 29 & Smólsko & 50.48228 & 22.78005 & 2004 \\
\hline & 78 & Korhynie & 50.40955 & 23.53079 & 2006 \\
\hline & 88 & Żurawce & 50.38259 & 23.54439 & 2005 \\
\hline \multirow[t]{3}{*}{ FB } & 20 & Gromada & 50.56583 & 22.70347 & 2004 \\
\hline & 21 & Kąty & 50.65225 & 22.72103 & 2004 \\
\hline & 22 & Żurawie & 50.8008 & 22.78019 & 2007 \\
\hline \multirow[t]{3}{*}{ FD } & 49 & Jurowce & 53.1929 & 23.15499 & 2006 \\
\hline & 59 & Krasne & 53.19637 & 23.29969 & 1983 \\
\hline & 59 & Krasny Las & 53.17361 & 23.3375 & 1983 \\
\hline
\end{tabular}




\begin{tabular}{llcccc}
\hline & 69 & Surażkowo & 53.21168 & 23.45654 & 1983 \\
FE & 21 & Knyszyn & 53.32651 & 22.92031 & 2006 \\
& 39 & Cimochowizna & 54.05896 & 23.0679 & 1991 \\
& 49 & Żubrówka & 54.0813 & 23.15286 & 1991 \\
& 51 & Machnacz & 53.33075 & 23.31183 & 1983 \\
& 51 & Wilcza Jama & 53.35416 & 23.30861 & 1983 \\
& 52 & Trzcianka & 53.46701 & 23.35709 & 1983 \\
XU & 04 & Stobnica & 52.70706 & 16.60989 & 2003 \\
\hline
\end{tabular}

Table 17. List of the northern birch mouse (Sicista betulina) localities.

\begin{tabular}{|c|c|c|c|c|c|}
\hline \multicolumn{2}{|c|}{ UTM square } & \multirow{2}{*}{$\begin{array}{c}\text { Localities } \\
\text { Kupin }\end{array}$} & \multicolumn{2}{|c|}{ Geographic coordinates } & \multirow{2}{*}{$\frac{\text { Year of capture }}{1992,1993,1995,1999}$} \\
\hline $\mathrm{DE}$ & 16 & & 53.84417 & 19.63652 & \\
\hline & 19 & Kajmy & 54.08861 & 19.72195 & 1992,1993 \\
\hline & 19 & Pasłęk & 54.06683 & 19.66181 & 1995,1999 \\
\hline & 19 & Winiary & 54.1255 & 19.71557 & 1993 \\
\hline & 44 & Elgnówko & 53.62721 & 20.21818 & 1988 \\
\hline & 45 & Łęguty & 53.75316 & 20.16362 & 1990 \\
\hline & 62 & Łyna & 53.43927 & 20.40728 & $\begin{array}{l}2000,2001,2002, \\
2003\end{array}$ \\
\hline & 72 & Natać Wielka & 53.51127 & 20.57244 & $\begin{array}{c}2000,2001,2002, \\
2007\end{array}$ \\
\hline & 72 & Natać Wielka Nowa & 53.51115 & 20.57941 & 2002 \\
\hline & 72 & Natać Wielka Stara & 53.50872 & 20.57132 & 2003 \\
\hline & 72 & Zimna Woda & 53.45215 & 20.6002 & 2002 \\
\hline & 73 & Bałdy & 53.59909 & 20.60773 & 2000 \\
\hline & 73 & Czarny Piec & 53.5472 & 20.62372 & $2000,2005,2007$ \\
\hline & 73 & Zgniłocha & 53.56399 & 20.56234 & $\begin{array}{c}2003,2004,2005, \\
2007\end{array}$ \\
\hline & 76 & Barczewko & 53.84854 & 20.59041 & 2003,2004 \\
\hline & 84 & Michałki & 53.65148 & 20.75707 & 2006 \\
\hline & 84 & Miluki & 53.66768 & 20.76514 & 2006 \\
\hline & 84 & Tylkowko & 53.63572 & 20.74358 & 2006 \\
\hline & 91 & Omulew & 53.42187 & 20.86719 & 2001 \\
\hline DF & 90 & Wardomy & 54.20512 & 20.90246 & 1987 \\
\hline DV & 15 & Polana Biały Potok & 49.27783 & 19.86588 & 2007 \\
\hline \multirow[t]{2}{*}{$\mathrm{EC}$} & 51 & Rokitnia & 51.61845 & 21.80539 & 1998 \\
\hline & 51 & Stężyca & 51.58256 & 21.77271 & 1997 \\
\hline \multirow[t]{4}{*}{$\mathrm{EE}$} & 43 & Anuszewo & 53.52396 & 21.68842 & 2003 \\
\hline & 43 & Wiartel & 53.59362 & 21.67953 & 2004 \\
\hline & 53 & Pogubie & 53.55816 & 21.76566 & 2003 \\
\hline & 79 & Czerwony Dwór & 54.1301 & 22.18694 & 1984 \\
\hline FB & 22 & Radecznica & 50.75113 & 22.83009 & 2007 \\
\hline \multirow[t]{4}{*}{ FD } & 39 & Fasty & 53.17171 & 23.04513 & 2000 \\
\hline & 49 & Jurowce & 53.19661 & 23.15286 & $\begin{array}{c}1993,1996,1999, \\
2000\end{array}$ \\
\hline & 59 & Krasne & 53.19637 & 23.29969 & 1983 \\
\hline & 59 & Krasny Las & 53.17361 & 23.3375 & 1983 \\
\hline
\end{tabular}




\begin{tabular}{cccccc}
\hline FD & 39 & Fasty & 53.17171 & 23.04513 & 2000 \\
& 49 & Jurowce & 53.19661 & 23.15286 & $1993,1996,1999$, \\
& & & & 2000 \\
& 59 & Krasne & 53.19637 & 23.29969 & 1983 \\
& 59 & Krasny Las & 53.17361 & 23.3375 & 1983 \\
& 59 & Podsupraśl & 53.20632 & 23.33534 & 1983 \\
& 68 & Żednia & 53.0901 & 23.46989 & 1983 \\
& 69 & Surażkowo & 53.21168 & 23.45654 & 1983 \\
FE & 40 & Karczmisko & 53.29976 & 23.18604 & 1983 \\
& 48 & Czerwony Krzyż & 54.0143 & 23.1571 & 2001 \\
& 49 & Maćkowa Ruda & 54.05129 & 23.17604 & $1995,1996,2001$ \\
& 50 & Budzisk & 53.24332 & 23.34981 & 1983 \\
& 51 & Machnacz & 53.33075 & 23.31183 & 1983 \\
& 51 & Wilcza Jama & 53.35416 & 23.30861 & 1983 \\
& 52 & Trzcianka & 53.46701 & 23.35709 & 1983 \\
60 & Sokołda & 53.24722 & 23.46676 & 1983 \\
\hline
\end{tabular}

Table 18. List of the forest dormouse (Dryomys nitedula) localities.

\begin{tabular}{cccccc}
\hline \multicolumn{2}{c}{ UTM square } & Localities & \multicolumn{2}{c}{ Geographic coordinates } & Year of capture \\
\hline FE & 61 & $\begin{array}{c}\text { Rezerwat Stara } \\
\text { Dębina }\end{array}$ & 53.315071 & 23.527018 & 1993 \\
& 40 & Karczmisko & 53.29976 & 23.18604 & 1983 \\
\hline
\end{tabular}

Table 19. List of the common dormouse (Muscardinus avellanarius) localities.

\begin{tabular}{cccccc}
\hline \multicolumn{2}{c}{ UTM square } & Localities & \multicolumn{2}{c}{ Geographic coordinates } & Year of capture \\
\hline DE & 19 & Kajmy & 54.08861 & 19.72195 & 1992 \\
DV & 15 & Polana Biały Potok & 49.27783 & 19.86588 & 2007 \\
FA & 88 & Plebanka & 50.40429 & 23.66052 & 2005 \\
FE & 53 & Romanówka & 53.536633 & 23.324072 & 1983 \\
\hline
\end{tabular}




\section{DANE O ROZMIESZCZENIU WYBRANYCH GATUNKÓW SORICOMORPHA I RODENTIA W POLSCE}

\section{STRESZCZENIE}

W niniejszej pracy przedstawiliśmy dane dotyczące występowania czterech gatunków Soricomorpha i piętnastu gatunków Rodentia. Dane o występowaniu zostały zgromadzone podczas odłowów ryjówek aksamitnych i myszy leśnych wielkookich w celu analiz zmienności chromosomowej, oraz chomika europejskiego w celu analiz filogeograficznych i zmienności genetycznej. Mapy występowania poszczególnych gatunków zostały wykonane w ArcGIS. Każdej mapie towarzyszą listy lokalizacji wraz ze współrzędnymi geograficznymi i rokiem odłowu.

\section{REFERENCES}

BANASZEK A., JADWISZCZAK K.A. 2006: B-chromosomes behaviour during meiosis of yellow-necked mouse, Apodemus flavicollis. Folia Zool., 55: 113-122.

Banaszek A., Jadwiszczak K.A., Ratkiewicz M., Ziomek J., Neumann K. 2010: Population structure, colonization processes and barriers for dispersal in the common hamster Cricetus cricetus (L.) populations in Poland. J. Zool. Syst. Evol. Res., 48:151-158.

Bialas I., Chętnicki W., Kupryjanowicz J. 1982: The dormice (Gliridae) in the Knyszyńska Forest. Prz. Zool., 26: 465-469 [in Polish].

Engel W., Vogel W., Voiculescu I., Ropers H., Zenzes M.T., Bender K. 1973: Cytogenetic and biochemical differences between Apodemus sylvaticus and Apodemus flavicollis possibly responsible for the failure to interbreed. Comp. Biochem. Physiol., 44B: 1165-1173.

FEDYK S., 1995: Geographic chromosomal differentiation and hybrid zones between chromosome races of Sorex araneus in north-eastern Poland. Dissertationes Universitatis Varsoviensis 439, Białystok : 1-125 [in Polish].

Fedyk S., Chętnicki W., Ruprecht A.L., Cichocki J., 2011: Chromosome polymorphism in Polish populations of northern birch mouse Sicista betulina. Folia Zool., 60: 31-36.

Mooney H.A., Cleland E.E. 2001: The evolutionary impact of invasive species. Proc. Nat. Acad. Sci., 98: 5446-5451.

Okarma H. 2009: The Atlas of Polish Mammals. In: Abstracts of XI International Theriological Conference, Department of Systematic Zoology, UAM, p 15 [in Polish].

Pucek Z., Raczyński J. /eds/, 1983: Atlas of Polish Mammals. Polish Scientific Publishers. Warszawa: 1-188.

Root T.L., Price J.T., Hall K.R., Schneider S.H., Rosenzweig C., Pounds J.A. 2003: Fingerprints' of global warming on animals and plants. Nature, 421: 57-60.

Willis K.J., Niklas K.J. 2004: The role of Quaternary environmental change in plant macroevolution: the exception or the rule? Phil. Trans. Roy. Soc., London B, 359: $159-172$.

Ziomek J., BanAsZeK A., 2007: The common hamster, Cricetus cricetus in Poland: status and current range. Folia Zool., 56: 235-242.

Received 2012-05-18 